\title{
ARTICLE
}

Genomics and Gene Therapy

\section{Base-edited CAR T cells for combinational therapy against T cell malignancies}

\author{
Christos Georgiadis $\mathbb{1}^{1}$ - Jane Rasaiyaah ${ }^{1}$ - Soragia Athina Gkazi ${ }^{1} \cdot$ Roland Preece $^{1}$ - Aniekan Etuk ${ }^{1}$ • \\ Abraham Christi ${ }^{1} \cdot$ Waseem Qasim $\mathbb{1}^{1,2}$
}

Received: 16 August 2020 / Revised: 20 March 2021 / Accepted: 4 May 2021

(c) The Author(s) 2021. This article is published with open access

\begin{abstract}
Targeting $\mathrm{T}$ cell malignancies using chimeric antigen receptor (CAR) $\mathrm{T}$ cells is hindered by ' $\mathrm{T} v \mathrm{~T}$ ' fratricide against shared antigens such as CD3 and CD7. Base editing offers the possibility of seamless disruption of gene expression of problematic antigens through creation of stop codons or elimination of splice sites. We describe the generation of fratricide-resistant T cells by orderly removal of TCR/CD3 and CD7 ahead of lentiviral-mediated expression of CARs specific for CD3 or CD7. Molecular interrogation of base-edited cells confirmed elimination of chromosomal translocations detected in conventional Cas9 treated cells. Interestingly, 3CAR/7CAR co-culture resulted in 'self-enrichment' yielding populations 99.6\% TCR-/CD3-/CD7-. 3CAR or 7CAR cells were able to exert specific cytotoxicity against leukaemia lines with defined $\mathrm{CD} 3$ and/or CD7 expression as well as primary T-ALL cells. Co-cultured 3CAR/7CAR cells exhibited highest cytotoxicity against CD3 + CD7 + T-ALL targets in vitro and an in vivo human:murine chimeric model. While APOBEC editors can reportedly exhibit guide-independent deamination of both DNA and RNA, we found no problematic 'off-target' activity or promiscuous base conversion affecting CAR antigen-specific binding regions, which may otherwise redirect $\mathrm{T}$ cell specificity. Combinational infusion of fratricide-resistant anti-T CAR T cells may enable enhanced molecular remission ahead of allo-HSCT for T cell malignancies.
\end{abstract}

\section{Introduction}

T cell acute lymphoblastic leukaemia (T-ALL) arises from lymphoid precursors and is typically associated with poorer prognosis to comparable B cell leukaemia. Standard therapy for T-ALL, uses high intensity multi-agent chemotherapy

These authors contributed equally: Christos Georgiadis, Jane Rasaiyaah.

The views expressed are those of the author(s) and not necessarily those of the NHS, the NIHR, or the Department of Health.

Supplementary information The online version contains supplementary material available at https://doi.org/10.1038/s41375021-01282-6.

Waseem Qasim

W.Qasim@UCL.ac.uk

1 Molecular and Cellular Immunology Unit, UCL Great Ormond Street Institute of Child Health, WC1N 1EH London, UK

2 NIHR Great Ormond Street Hospital Biomedical Research Centre, 30 Guilford Street, London, UK where tolerated and sensitive minimal residual disease assessments can determine when molecular remission is achieved. However, 5 year survival for T-ALL is $<75 \%$ for children and $<50 \%$ for adults [1] and while salvage therapy such as nelarabine [2] and allogeneic stem cell transplantation (SCT) [3] are widely used, innovative therapies have lagged behind progress for other leukaemias. Strategies using chimeric antigen receptor (CAR) $\mathrm{T}$ cells are being widely applied against $\mathrm{B}$ cell malignancies, and targeting of surface antigens such as CD19, CD20 and CD22, which are also present on healthy B cells, has been shown to be broadly tolerable [4-6]. A proportion of patients with sustained B cell aplasia require long term immunoglobulin replacement, and some may go on to allogeneic transplant and donor derived immunological recovery $[7,8]$. Similar approaches to tackle $\mathrm{T}$ cell malignancies have been more challenging because surface expression of surface antigens such as the TCR $\alpha \beta / \mathrm{CD} 3$ complex results in compromising fratricidal effects during $\mathrm{T}$ cell production. We have previously reported how this issue can be circumvented by removal of cell surface CD3 through disruption of TRAC expression by TALEN genome editing [9]. Therapeutic applications envisaged strictly time-limited 
effector activity to secure remission, and then rapid reconstitution of a diverse $\mathrm{T}$ cell compartment given that substitution of $\mathrm{T}$ cell function (unlike $\mathrm{B}$ cell function) is not possible. The kinetics of autologous $\mathrm{T}$ cell recovery in such settings has yet to be defined but could take many months and may be limited to post thymic populations with restricted TCR repertoire diversity. The availability of a Human Leucocyte Antigen (HLA)-matched allogeneic donor may offer the prospect of donor derived $\mathrm{T}$ cell recovery, but in either setting the strategy relies on first securing rapid and deep molecular remission through CAR $\mathrm{T}$ effects. The additional issue of antigen escape or incomplete expression on disease populations has resulted in a quest for additional antigens, and proposals to target multiple antigens simultaneously. Similar approaches are under development for B cell malignancies, for example by using CAR T cells targeting CD19 and CD22 or CD123 generated using bicistronic constructs or tandem CAR configurations [10-12]. Alternatively, a more physiological and flexible strategy involves the generation of multiple $\mathrm{T}$ cell banks with differing CAR specificities to be used in combination, as determined by particular disease specific cell surface molecule expression. We have investigated CAR $\mathrm{T}$ cells specific for $\mathrm{CD} 3$ and $\mathrm{CD} 7$ for combinational use against $\mathrm{T}$ cell malignancies. The latter is a $40-\mathrm{kD}$ singledomain Ig superfamily transmembrane glycoprotein encoded on chromosome 17q25.3 and highly expressed on normal and malignant $\mathrm{T}$ cells. Others have previously generated anti-CD7 CAR T cells following expression of inhibitory proteins [13] or CRISPR/Cas9 [14-16] editing of T cells to avoid fratricidal effects. We addressed the risk of fratricide and crossrecognition during co-culture of anti-CD3 and anti-CD7 CAR effectors by removal of cell surface targets recognised by the respective CARs. To achieve this efficiently, and with reduced risk of translocations, multiplexed base editing was combined with lentiviral CAR delivery. Base editing used CRISPR guided chemical deamination for highly precise, seamless, cytidine to uridine to thymidine $(\mathrm{C} \rightarrow \mathrm{U} \rightarrow \mathrm{T})$ conversion which then directed the creation of stop codons or disrupted splice donor/acceptor sites. The technology used $\mathrm{BE} 3$ [17], comprising a deactivated Cas9 nickase fused to rat APOBEC1 (the deaminase) and a single uracyl glycosylase inhibitor (UGI) delivered as mRNA by electroporation in combination with CRISPR guides. Highly specific RNA guided cytidine deamination in $\mathrm{T}$ cells ahead of lentiviral transduction ensured target antigen disruption before CAR expression. An important consideration in the context of multiplexed editing to date has been the creation of chromosomal translocations following nuclease-mediated double strand breakage, especially when multiple genomic loci are targeted simultaneously. Previously, we had reported that around 5\% of TALEN edited CAR19 T cells, disrupted at both TRAC and CD52 loci, exhibited karyotypic abnormalities [18-20], and a similar frequency was recently reported in
CRISPR/Cas9 edited T cells expressing recombinant TCR [21]. Advantages of base editing were expected to include high efficiency editing [22] and a notable reduction of otherwise predictable translocations between edited chromosomes. We report that removal of shared antigens permits generation of anti-CD3 and anti-CD7 CAR T cells and enables combinational effector activity against $\mathrm{T}$ cell targets. The strategies are readily scalable through the adaption of existing semi-automated manufacturing processes. It is envisaged that a time limited therapeutic application of combinational anti-T CAR $\mathrm{T}$ cells could deliver deep molecular remission ahead of conditioning and programmed allogeneic SCT in difficult to treat T-ALL.

\section{Results}

\section{Combinational CAR T cell targeting of CD3 and CD7 requires multiplexed base conversion for simultaneous removal of shared antigens}

We have previously described a second generation chimeric antigen receptor, $3 \mathrm{CAR}$, specific for $\mathrm{CD} 3 \varepsilon$ comprising an $\mathrm{scFv}$ sequence derived from the therapeutic monoclonal antibody OKT3 fused to a CD8 hinge and transmembrane domain and 41BB-CD3 $\zeta$ endodomain [9]. TALEN mediated genetic disruption of the TRAC locus had ensured that T cells subsequently transduced with a lentiviral-CD3 CAR targeting vector lacked cell surface expression of TCR $\alpha \beta / \mathrm{CD} 3$ and thereby evaded fratricidal effects. Similar effects were also readily elicited using CRISPR directed Cas9 editing, and investigations revealed the critical importance of orderly disruption of the target antigen locus ahead of CAR delivery and expression. The same principles were applied to evaluate a CAR specific for CD7 (Fig. 1A-C). The construct was generated using light and heavy variable domains derived from the 3A1e hybridoma as previously reported [15] and fused to a CD8TM-41BB-CD3 $\zeta$ configuration within a third generation SIN lentiviral vector (Fig. 1B).

Genome editing at multiple loci was applied to prevent fratricide during co-culture using sgRNA guides against TRBC1/2 and CD7 capable of operating with both CRISPR/Cas9 and cytidine base editing (BE) reagents (Fig. 1C). Thus stabilised guides with 2'-O-methyl $3^{\prime}$ phosphorothioate modifications were electroporated along with CleanCap SpCas9 mRNA $\sim 24 \mathrm{~h}$ after activation with anti-CD3/CD28 antibodies.

Transient RNA mediated expression of editing reagents was designed to ensure dilutional elimination in dividing cells for reduced off-target effects and immunogenicity. After a further $24 \mathrm{~h}$, lentiviral 3CAR or 7CAR transduction (MOI 5) was followed by 12 days of culture. High level surface disruption of TCR $\alpha \beta / \mathrm{CD} 3$ and $\mathrm{CD} 7$ was confirmed 


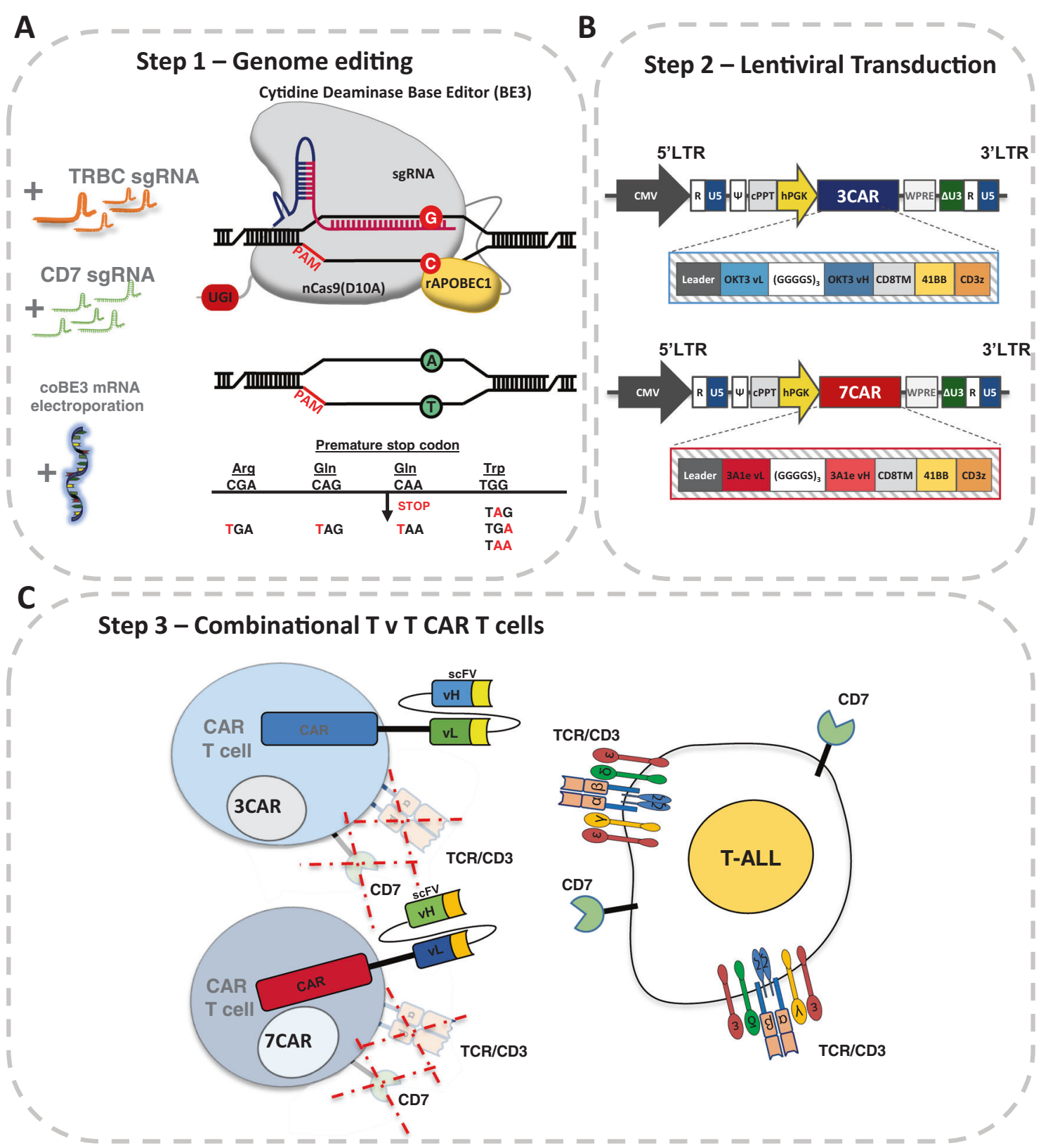

Fig. 1 Generation of ' $T$ v $T$ ' fratricide resistant CAR $T$ cells. A Schema of base editing for $\mathrm{T}$ cells employing 3rd generation codon optimised cytidine base deaminase (coBE3) fused to deactivated D10A Cas9 nickase and uracil glycosylase inhibitor (UGI) delivered as mRNA along with TRBC and CD7 single guide RNA (sgRNA). C$>\mathrm{U}->\mathrm{T}$ conversion (G->A antisense strand) resulting in STOP codon. B Lentiviral transduction of edited cells from step 1 using 3rd generation lentiviral vectors. Lentiviral plasmid configuration of CD3 $\varepsilon$ targeting 2nd generation chimeric antigen receptor comprising OKT3 $\mathrm{vL}$ and $\mathrm{vH} \mathrm{scFv}$ sequence fused to CD8 transmembrane domain (TM), 41BB co-stimulatory and $\mathrm{CD} 3 \mathrm{z}$ activation domains under the control of a hPGK promoter. Lentiviral plasmid configuration of CD7

by flow cytometry (63.7-97.9\% and 60.8-95.6\%, respectively) using SpCas9 mRNA and was associated with robust CAR expression throughout culture in multiple donors $(n=$ 4) (Supplementary Figs. 1A, B and 2A). Application of targeting 2nd generation CAR comprising 3A1e vL and $\mathrm{vH} \mathrm{scFv}$ sequence fused to CD8TM-41BB-CD3z under the control of a hPGK. $\mathrm{C}$ coBE3 edited $\mathrm{T}$ cells devoid of shared antigens TCR/CD3 and CD7 surface receptors expressing either 3CAR or 7CAR evade fratricide and target T-ALL. BE: base editor; APOBEC: (apolipoprotein B mRNA editing enzyme, catalytic polypeptide-like); sgRNA: single guide RNA; PAM: protospacer adjacent motif; LTR: long terminal repeat; CMV: cytomegalovirus promoter; CAR: chimeric antigen receptor; cPPT: central polypurine track; U5: untranslated $5^{\prime}$ region; DU3: delta untranslated $3^{\prime}$ region; hPGK: human phosphoglycerate kinase promoter; vL: variable light chain; $\mathrm{vH}$ : variable heavy chain.

coBE3 mRNA mediated similar levels of disruption of cell surface expression of TCR $\alpha \beta / \mathrm{CD} 3(63.4-96.2 \%)$ and CD7 (49.3-72.6\%) with double knockout accounting for $37.5-61.8 \%$ of cells, comparable to SpCas9 treated cells 

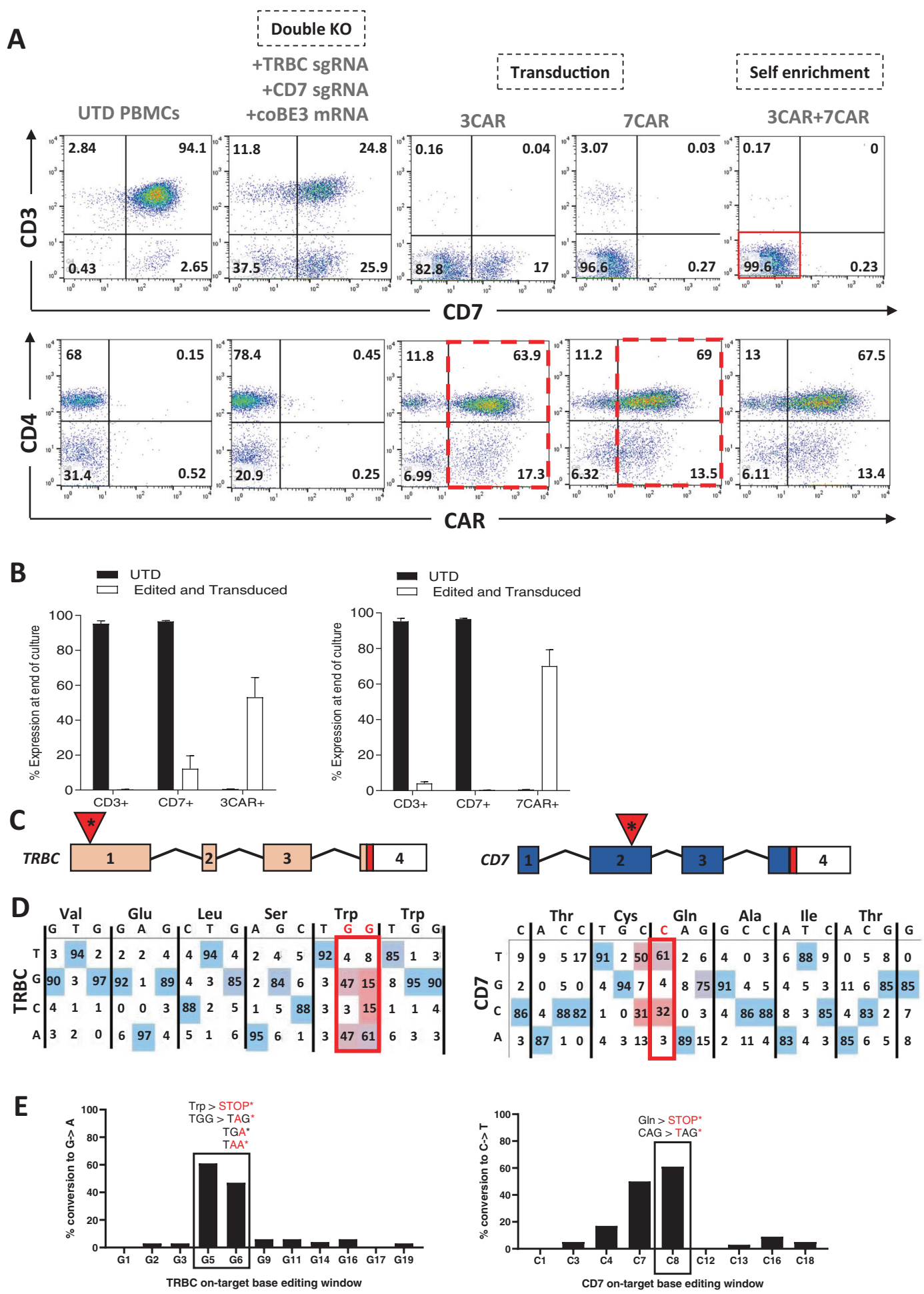

$(P=0.2)$ (Fig. 2A, B and Supplementary Figs. 1A, B and 2A). On-target genome editing in coBE3 edited cells was verified at the molecular level at $61-100$ and $42-71 \%$ (G > A conversion) for positions $\mathrm{G}_{5} \mathrm{G}_{6}$ in TRBC, and $61-94 \%$ conversion of $\mathrm{C}>\mathrm{T}$ for position $\mathrm{C}_{8}$ in $\mathrm{CD} 7$ (Fig. $2 \mathrm{C}$, D and $\mathrm{E}$ and Supplementary Fig. 2B) within the expected $5 \mathrm{bp}$ windows of predicted BE activity. Beyond the TRBC target

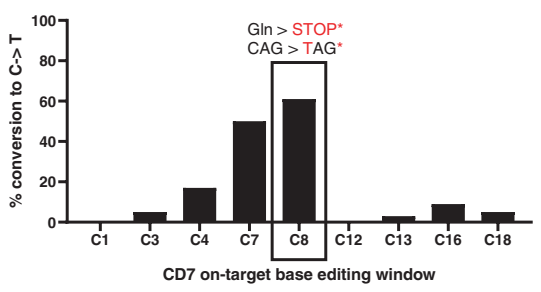

window, there were $\leq 6 \% \mathrm{G}>\mathrm{A}$ conversions of guanine nucleotides, and $\leq 9 \% \mathrm{C}>\mathrm{T}$ conversions outside the predicted $5 \mathrm{bp}$ window for CD7 (Fig. 2D, E). Neither SpCas9 editing nor cytidine deamination impacted on CAR expression with comparably high level 3CAR (SpCas9: 32.3-78.2\%; coBE3: 43.7-81.2\%) and 7CAR (SpCas9: 39.9-80.51\%; coBE3: 48.7-82.5\%) expression at the end of 
Fig. 2 3CAR and 7CAR primary $T$ cells evade fratricide during production. A Phenotypic analysis of surface antigen $\mathrm{CD} 3$ and $\mathrm{CD} 7$ expression (top panel) and CAR expression (bottom panel) of CD3/ CD28 activated peripheral blood mononuclear cells elcetroporated with sgRNA targeting TRBC and CD7 alongside coBE3 mRNA and subsequently transduced with 3CAR or 7CAR lentiviral vectors at MOI 5. Reduced TCR/CD3 and CD7 expression in edited groups and high level CAR expression with fratricide evasion (dotted red outline) was exhibited $(n=4)$. Self-enrichment effects followed co-culture of $3 \mathrm{CAR}$ and 7CAR products $(n=2)$ resulted in enriched $\mathrm{TCR}^{-} \mathrm{CD}^{-}$ 3CAR/7CAR cells (red box). B Proportion of CD3 or CD7 surface antigen and CAR expression at end of 3CAR $(n=4)$, 7CAR $(n=4)$ production or untransduced (UTD) $(n=4)$ cells. Error bars represent SEM across $(n=4)$ donors. C Schematic of exonic regions within $T R B C$ and $C D 7$ genes. Red marking in exons 4 of TRBC and $C D 7$ represent genomic translation stop sites followed by $5^{\prime}$ untranslated regions (white boxes). Red triangles with asterisk indicate position of base conversion resulting in premature stop codon formation. D Representative base EDITR output of Sanger sequencing results from mixed 3CAR/7CAR co-culture DNA PCR amplicons of TRBC and $C D 7$ genomic loci. Sites of intended base conversion highlighted in red boxes. High frequency $\mathrm{G}>\mathrm{A}$ (antisense) and $\mathrm{C}>\mathrm{T}$ changes within editing window highlighted by red vs blue colour. $\mathbf{E}$ Percentage of $\mathrm{G}>\mathrm{A}$ conversions throughout TRBC-targeting protospacer sequence (left), and $\mathrm{C}>\mathrm{T}$ conversions throughout $\mathrm{CD}$ 7-targeting protospacer sequence (right).

production (Fig. 2A, B and Supplementary Figure. 1A). Importantly, there were no differences in cell expansion and final cell yields generated from primary $\mathrm{T}$ cell donors using Cas9 or coBE3 (Supplementary Figure. 3). Phenotype profiles assigned to naïve, central memory and effector memory populations were determined on both $\mathrm{CD}^{+}$and $\mathrm{CD} 8^{+}$CAR $\mathrm{T}$ cells for base edited and SpCas9 modified cells (Supplementary Fig. 4). By the end of culture all groups exhibited exhaustion markers PD-1, TIM-3 or LAG3 at levels comparable to baseline, with no detectable differences between SpCas9 and coBE3 treated cells (Supplementary Fig. 5).

Co-culture of edited 3CAR and 7CAR products demonstrated the critical advantage of shared antigen removal from both products yielding a $97.8-99.6 \% \mathrm{TCR} \alpha \beta^{-} \mathrm{CD} 7^{-}$population expressing high levels of 3CAR/7CAR (SpCas9: 37.6-78.4\%; coBE3: 49.1-80.9\%) suggesting evasion of fratricide effects (Fig. 2A and Supplementary Fig. 1A and 2A). Interestingly, co-culture also allowed self-enrichment effects by eliminating residual $\mathrm{CD}^{+}$or $\mathrm{TCR} \alpha \beta^{+}$cells, although further TCR $\alpha \beta$ magnetic column-based depletion may be warranted to ensure stringent depletion of residual TCR $\alpha \beta$ cells in the allogeneic setting (Fig. 2A, B and Supplementary Fig. 1A and 2A).

\section{Cytidine base editing reduces predicted dsDNA chromosomal translocations}

DSBs can lead to chromosomal translocations between sites of multiplexed editing, and this has been reported in
TALEN and CRISPR/Cas9 modified T cells. Base conversion offers the prospect of seamlessly disrupting TRBC1/2 and $C D 7$ without DSBs and thus we examined genomic DNA at the end of production 3CAR and 7CAR primary $\mathrm{T}$ cells following multiplexed TRBC1/2 and CD7 editing and compared the frequency of predicted translocations generated by SpCas9 or coBE3 activity. DNA fragments bearing each of four predicted fusions between two disrupted chromosomal loci were synthesised to provide control readings for PCR reactions across the novel junctions (Fig. 3A). Using a combination of TRBC1/2 and CD7 binding primers, PCR, was able to detect signals in $3 / 4$ sites for SpCas9 (T1, T2, T3) and 1/4 (T1) sites for coBE3 treated samples (Fig. 3B). Upon further investigation, ddPCR quantified translocations detected for SpCas9 edited samples of $0.25-0.98 \%$ for all four combinations (Fig. 3) whereas only very low level events $(<0.18 \%)$ were detected for $\mathrm{T} 1$ and $\mathrm{T} 3$ in coBE3 edited cells (Fig. 3C).

\section{CAR \& 7CAR T cell cytotoxicity}

In vitro function of 3CAR and 7CAR effector T cells was initially assessed by co-culture experiments with ${ }^{51} \mathrm{Cr}$ labelled Jurkat $\mathrm{T}$ cell targets, either alone or in combination. Alone, base edited 3CAR or 7CAR primary T cells exhibited high levels of cytotoxic activity against $\mathrm{CD} 3^{+} \mathrm{CD} 7^{+}$ targets, with both 3CAR and 7CAR effectors lysing between 40 and 70\% of targets at an E:T of 20:1 (Fig. 4A and Supplementary Fig. 6A). Cytotoxic activity was comparable to SpCas9-edited 3CAR or 7CAR cells indicating intact functional integrity after base editing (Supplementary Figs. 6B and 7). Against antigen-negative controls, coculture of either 3CAR or 7CAR effectors against $\mathrm{CD}^{-}$ $\mathrm{CD}^{-}$targets at an equivalent E:T ratio, resulted in $<5 \%$ cytotoxicity. Specificity was further corroborated by coculture of either 3CAR or 7CAR effectors with mixed population target cells, comprising either single positive $\mathrm{CD}^{+}{ }^{+} \mathrm{CD} 7^{-}$or, $\mathrm{CD}^{-} \mathrm{CD}^{+}$, or a heterogeneous mix of $\mathrm{CD}^{+/-} \mathrm{CD}^{+/-}$populations. This demonstrated CARspecific clearance was restricted to cells exhibiting their respective antigens, and absence of lysis of antigen negative cell fractions (Fig. 4A and Supplementary Figs. 6 and 8). In a clinical setting, combined infusions of 3CAR and 7CAR $\mathrm{T}$ cells may be required and when 3CAR T cells were cocultured with 7CAR T cells and then used in combination, they exhibited high level cytotoxicity against mixed antigen $\mathrm{CD}^{-}{ }^{-} \mathrm{CD} 7^{+}$and $\mathrm{CD}^{+}{ }^{+} \mathrm{CD}^{-}$populations with $>30 \%$ lysis at E:T ranges between 10:1 and 5:1 (Fig. 4A and Supplementary Figs. 6 and 8).

Next the function of 3CAR and 7CAR base edited T cells was assessed against primary paediatric $\mathrm{T}$ cell acute lymphoblastic leukaemia (T-ALL). Flow based characterisation of $n=7$ primary T-ALL samples indicated high levels of 


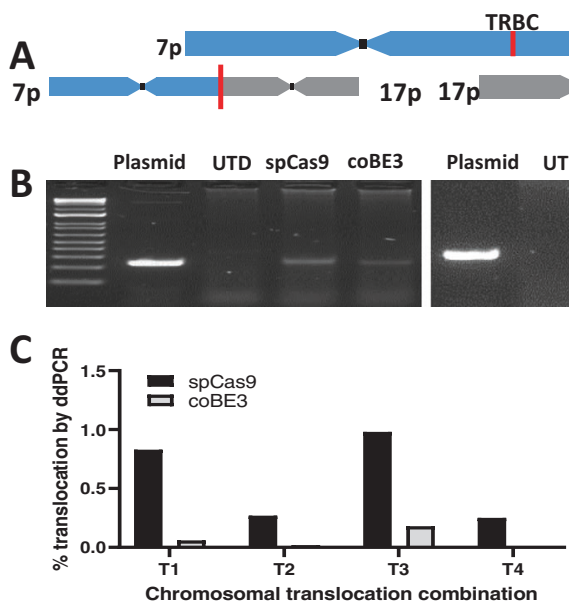

Fig. 3 Multiplexed cytidine deamination reduces frequency of dsDNA break-mediated chromosomal translocations. A Schematic of TRBC locus within chromosome $7 \mathrm{q}$-arm and $C D 7$ locus within chromosome 17 q-arm highlighted by red line. Four predicted chromosomal translocations generated following simultaneous dsDNAmediated cleavage at $T R B C$ and $C D 7$ loci. B Gel electrophoresis of DNA products from either untransduced (UTD) or mixed 3CAR/ 7CAR co-cultures edited with SpCas9 or coBE3 mRNA following

cell surface CD7 in these patient samples, ranging from 83 to $98 \%$. In contrast, both surface and intracellular expression of CD3 were highly variable $1.5-86$ and $2-92 \%$, respectively, again highlighting the likely need to target multiple antigens in these subjects. A T-ALL sample where 9.6\% of cells expressed surface CD3 and $92.8 \%$ expressed CD7, was loaded with fluorescent dye before culture with effector CAR T cells. Targets were depleted by 3CAR cells (residual $2.7 \% \mathrm{CD} 3$ ) or 7CAR cells (residual 3.3\% CD7) alone, and premixed 3CAR/7CAR $\mathrm{T}$ cells mediated almost complete elimination of target cells (Fig. 4B).

\section{Base edited 3CAR and 7CAR T cells exhibit potent anti-leukaemic effects in vivo}

To evaluate in vivo anti-leukaemic potency, edited 3CAR and 7CAR $\mathrm{T}$ cells were investigated in a previously described humanised NOD/SCID $/ \gamma \mathrm{c}^{-1-}$ (NSG) xenograft model of leukaemic T cell inhibition [9]. Mice $(n=28)$ were first infused with $\mathrm{EGFP}^{+} \mathrm{LUC}^{+}$labelled Jurkat T cells expressing CD3 and CD7 (Supplementary Fig. 9) and engraftment was confirmed by bioluminescent imaging 3 days later. 3CAR or 7CAR cells edited using either SpCas9 or coBE3 mRNA were infused intravenously on day 4 alongside PBS and untransduced controls and leukaemic progression was monitored by serial bioluminescent imaging on over four weeks (Fig. 5A, B). Independently, both 3CAR and 7CAR cells were able to rapidly clear $3^{+} 7^{+}$ Jurkat targets as early as day 7 and prevent leukaemia progression over the 31 day observation period. On the
PCR amplification with TRBC Fwd - CD7 Fwd, TRBC Rev - CD7 Fwd, TRBC Rev - CD7 Rev and TRBC Fwd - CD7 Rev primer combinations. Positive bands detected at $\sim 250 \mathrm{bp}$. Control bands are PCR amplicons from of synthesised fusions. C Histogram showing percentage of digital droplet PCR (ddPCR)-based quantification of four possible predicted translocations (T1-T4) in DNA from mixed 3CAR/7CAR co-cultures edited with SpCas9 or coBE3 mRNA.

contrary, untreated mice showed clear disease progression with a $>4-\log$ difference in radiance (Fig. 5B, C). Loss of bioluminescent signal in CAR effector treated mice was corroborated by flow analysis which confirmed clearance of Jurkats from bone marrow (Fig. 5D and Supplementary Fig. 10A, B). There was no significant difference observed in the anti-leukaemic potency of CAR $\mathrm{T}$ cells generated using SpCas9 compared to coBE3 mRNA. To demonstrate the advantage of shared antigen removal from the CAR $T$ cell surface $(n=8)$ mice were co-infused with $5 \times 10^{6}$ of each 3CAR and 7CAR effectors. Similar to individual CAR $T$ cell infusions, the combination of fratricide-resistant 3CAR/7CAR effectors was able to persist throughout the 31 day monitoring period to successfully eradicate the disease (Fig. 5 and Supplementary Fig. 10).

When CAR specific antigen was not present, mice $(n=17)$ engrafted with $\mathrm{EGFP}^{+} \mathrm{LUC}^{+}$Jurkats modified to be $3^{-} 7^{-}$, neither 3CAR nor 7CAR T cells delivered independently or in combination were able to inhibit leukaemia and all mice succumbed to disease by day 24 (Supplementary Fig. 11).

\section{Combinational effects of base edited anti-T cells in humanised mice}

Mice $(\mathrm{n}=27)$ were engrafted with $1 \times 10^{7} \mathrm{EGFP}^{+} \mathrm{LUC}^{+}$ labelled Jurkat $\mathrm{T}$ cells modified to express either $\mathrm{CD} 3$ or CD7, alone or in combination, (Supplementary Figure. 9) and 3 days later leukaemia establishment was confirmed by bioluminescent signalling (Fig. 6A). After 4 days, mice were injected with coBE3 edited 3CAR/7CAR T cells (after 
Fig. 4 3CAR/7CAR base edited $T$ cells mediate potent killing of T-ALL cells in vitro. In vitro cytotoxicity of base edited 3CAR and 7CAR cells against T-ALL cell lines and primary T-ALL targets. A ${ }^{51} \mathrm{Cr}$ labelled Jurkat $\mathrm{T}$ cells modified to express $\mathrm{CD}^{+} \mathrm{CD}^{+}, \mathrm{CD}^{+}$ $\mathrm{CD}^{-}, \mathrm{CD}^{-} \mathrm{CD}^{+}$or $\mathrm{CD} 3^{-}$ $\mathrm{CD}^{-}$were co-cultured with either 3CAR (white squares), 7CAR (grey squares), mixed 3CAR/7CAR (black squares) or untransduced (white circles) cells at an increasing ratio of effectors:targets (E:T). Error bars represent SEM of $(n=3)$ technical replicates. B Cytotoxic activity of 3CAR, 7CAR, mixed 3CAR/7CAR or untransduced primary $\mathrm{T}$ cell controls against primary patient T-ALL (T-ALL \#7) cells. Representative flow cytometry plots gated on $\mathrm{CFSE}^{+}$ live T-ALL tumour cells (top panel). Frequency of surface antigens CD3, CD7 (middle panel) and CD19, CD56, (lower panel) gated on CFSE + live Jurkat cells.
A
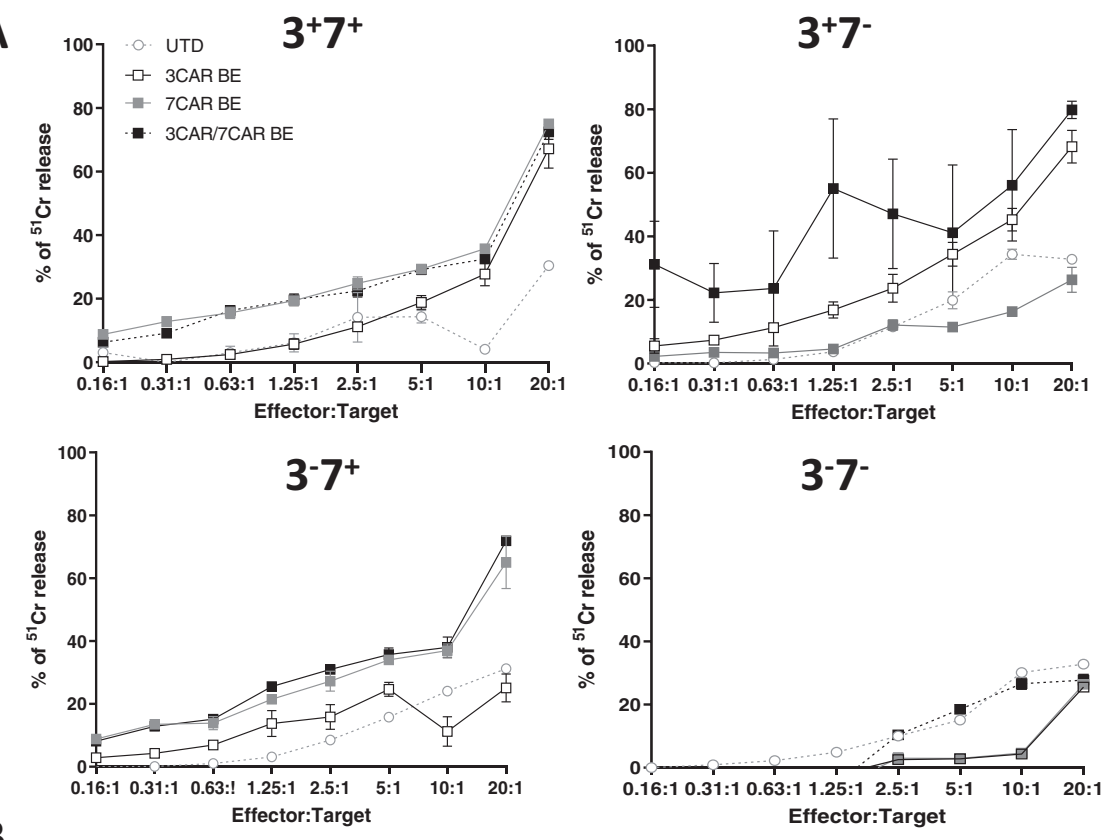

B
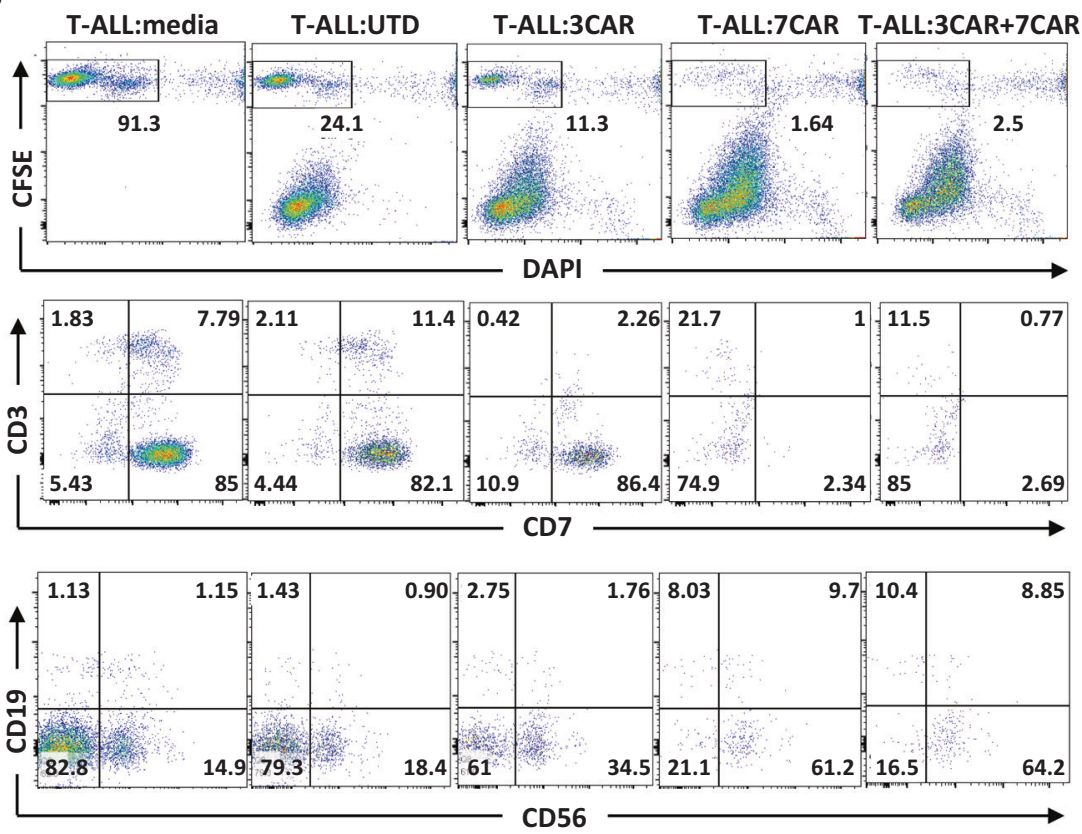

co-culture) or untransduced cells and leukaemic progression was monitored by serial bioluminescent imaging over 24 days (Fig. 6A, B). Within 7 days of 3CAR/7CAR effector infusion there was inhibition of luciferase signal in $3^{+} 7^{+}(\mathrm{n}=5), 3^{+} 7^{-}(\mathrm{n}=4)$ and $3^{-} 7^{+}(\mathrm{n}=5)$ target groups, but not in the $3^{-} 7^{-}(\mathrm{n}=5)$ cohort. By day 24 , leukaemic burden in the $3^{-} 7^{-}$infused group receiving 3CAR/7CAR effectors had increased $>400$-fold, comparable to leukaemic progression in the untransduced cohort (ns $P=0.36$ ) (Fig. 6B). This was further corroborated by bone marrow examination of $3^{-} 7^{-}$mice receiving mixed 3CAR/7CAR effectors with detection of marked expansion of
$\mathrm{GFP}^{+} \mathrm{CD} 2^{+}$Jurakts in mice receiving untransduced cells. In contrast, mice receiving premixed 3CAR/7CAR exhibited most complete eradication of $\mathrm{GFP}^{+} \mathrm{CD} 2^{+}$T-ALL cells expressing one or both surface antigens $\mathrm{CD} 3$ and $\mathrm{CD} 7$ (Fig. 6C, D).

\section{Guide dependent and independent off-target base editing effects}

The sgRNA guides adopted for $\mathrm{T}$ cell engineering exhibited high levels of on-target editing activity in combination with either SpCas9 or coBE3. We have previously described 
A
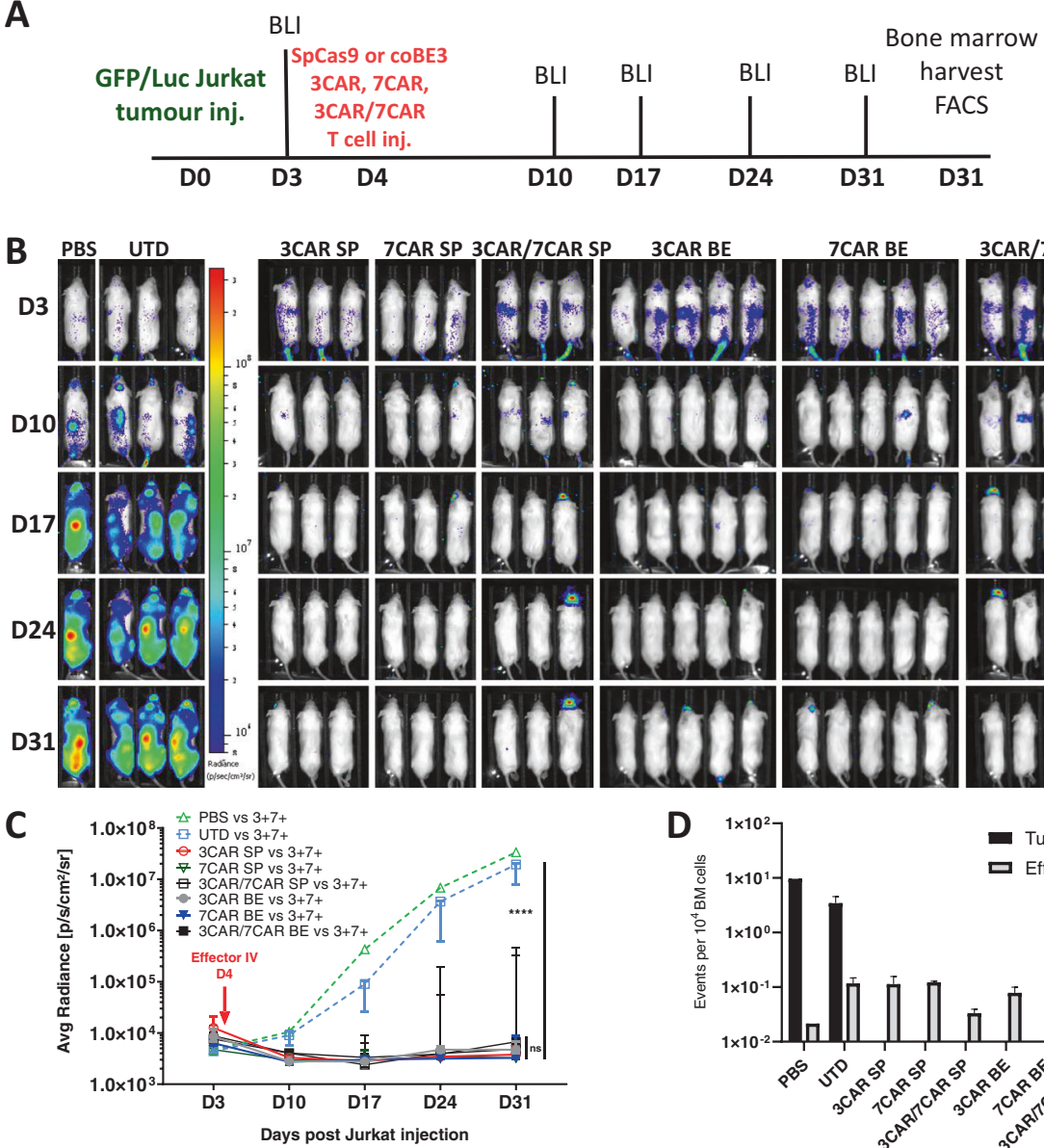

Fig. 5 Base edited 3CAR and 7CAR cells demonstrate comparable anti-tumour clearance in vivo to CRISPR-Cas9 edited cells. A Experimental timeline of $\mathrm{GFP}^{+} \mathrm{LUC}^{+}$Jurkat T cell injection (Day 0) and effector T cell injection (Day 4) in $n=28 \mathrm{NOD} / \mathrm{SCID} / \mathrm{cc}^{-1-}$ (NSG) mice. Bioluminescent imaging (BLI) performed biweekly (Days 3-31). Organ harvest post mortem for flow-based characterisation (Day 31). B $n=28 \mathrm{NOD} / \mathrm{SCID} / \mathrm{\gamma c}^{-/-}$(NSG) mice were intravenously injected with $10 \times 10^{6} \mathrm{GFP}^{+} \mathrm{LUC}^{+}$Jurkat $\mathrm{T}$ cells flow sorted for $\mathrm{CD}^{+} \mathrm{CD}^{+}$expression (Day 0) prior to receiving an IV infusion of effector T cells (Day 4). Mice received either $10 \times 10^{6}$ SpCas9 edited $\mathrm{TCR}^{-} \mathrm{CD}^{-}$3CAR $(n=3), 10 \times 10^{6}$ SpCas9 edited $\mathrm{TCR}^{-} \mathrm{CD}^{-}$ 7CAR $(n=3), 10 \times 10^{6}$ SpCas9 edited $\mathrm{TCR}^{-} \mathrm{CD}^{-}$3CAR/7CAR $(n=3), 10 \times 10^{6}$ coBE3 3 edited TCR-CD7 ${ }^{-} 3 \mathrm{CAR}(n=5), 10 \times 10^{6}$

strategies for investigating possible off-target guide mediated effects of SpCas9 using a combination of targeted sequencing of predicted sites, Digenome analysis, and whole-genome sequencing for signatures of NHEJ repair [23]. In the case of BE3, $\mathrm{C}>\mathrm{T}$ base conversion within a defined window has been previously quantified at the TRBC locus and NGS detected low-frequency NHEJ effects [24]. In addition reports of guide-independent BE activity both at the DNA and RNA level have been reported raising the prospect of promiscuous editing activity [2527]. Much of the published experience relates to experiments performed in cell lines following plasmid-mediated
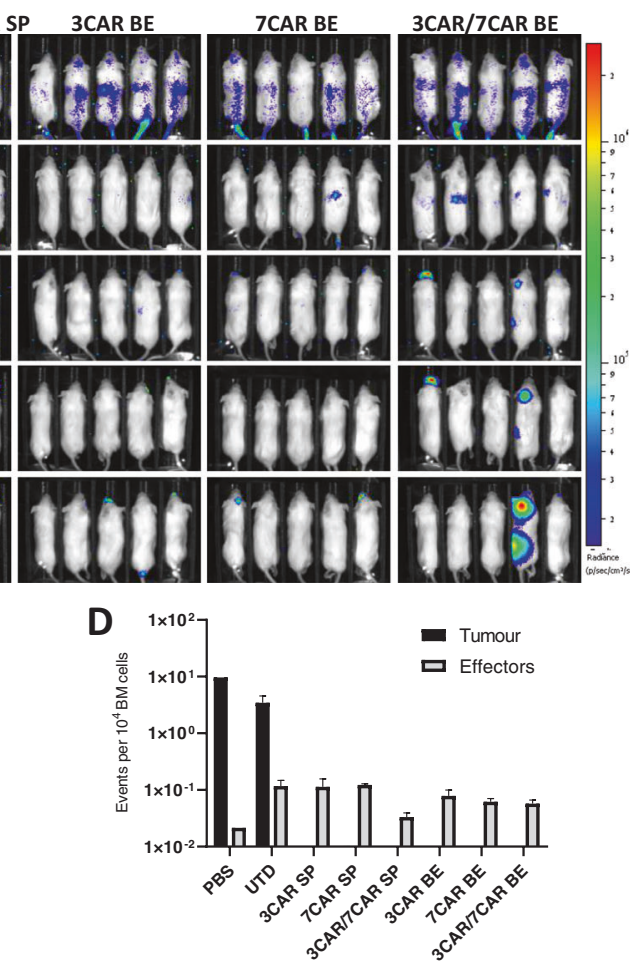

coBE3 edited $\mathrm{TCR}^{-} \mathrm{CD}^{-}$7CAR $(n=5)$, or $10 \times 10^{6}$ coBE3 edited $\mathrm{TCR}^{-} \mathrm{CD}^{-}$3CAR/7CAR $(n=5)$ effectors. Control mice received untransduced (UTD) cells $(n=3)$ or PBS $(n=1)$. Leukaemic progression was monitored by serial bioluminescent imaging (BLI) until day 31 when all groups were sacrificed. $\mathbf{C}$ Bioluminescence signal of each animal plotted as Average radiance [photons $/ \mathrm{s} / \mathrm{cm}^{2} / \mathrm{sr}$ ]. Each line represents a different experimental group and each point on the line the mean of each group. Error bars represent median with interquartile range. Area under the curve was calculated for each experimental group and values were compared using a one-way ANOVA with Tukey multiple comparison post-hoc. D Number of $\mathrm{CD}^{+} \mathrm{GFP}^{-}$effector events or $\mathrm{CD}_{2}^{+} \mathrm{GFP}^{+}$tumour events per $1 \times 10^{4}$ acquired bone marrow events. Error bars represent SEM. $* * * P<0.001 * * * * P<0.0001$.

expression of the editors and effects following mRNA mediated expression in primary cells may differ. Electroporation of BE mRNA into $\mathrm{T}$ cells was confirmed by Western blot to support transient protein expression $(<48 \mathrm{~h})$ and should reduce the likelihood of off-target editing (Supplementary Fig. 12).

NGS interrogation found 'on-target' base conversion frequencies were $>75 \%$ at both TRBC and $\mathrm{CD} 7$ sites in 3CAR BE and 7CAR BE T cells compared to $<0.9 \%$ in SpCas9 edited samples (Supplementary Fig. 13A, B). 'Ontarget' TRBC and CD7 NHEJ events manifesting as insertions/deletions (indels) were between $50-60 \%$ in SpCas9 
A

Effector

D3

D11

D17

D24

Target

过

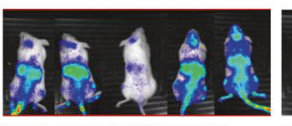
A1

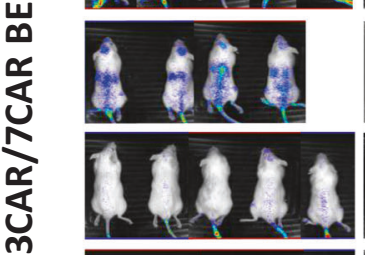
III

Mivi it II) 1812: 1111.

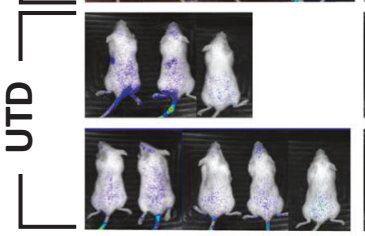
IN1 I0 A I I I Biti

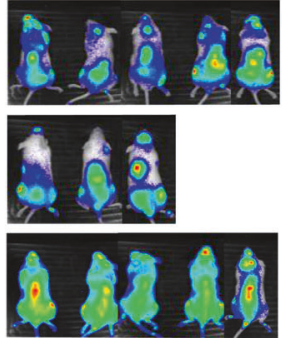
init oth
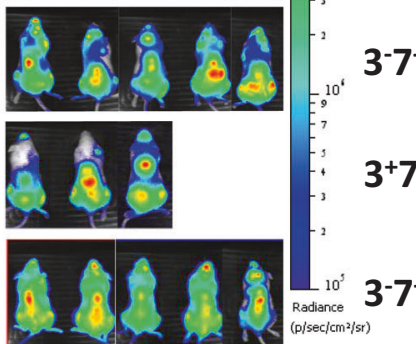

B

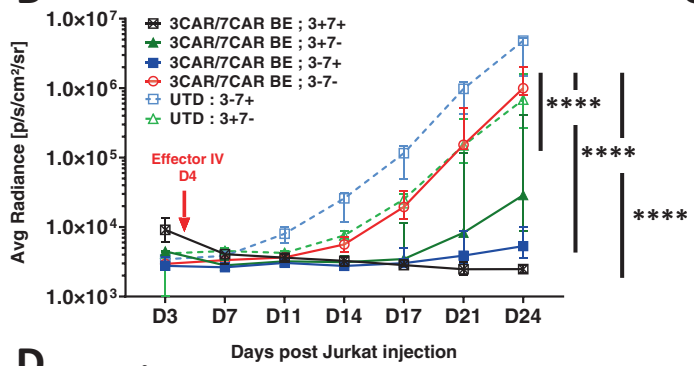

C 3CAR/7CAR BE :

3CAR/7CAR BE: 3CAR/7CAR BE:
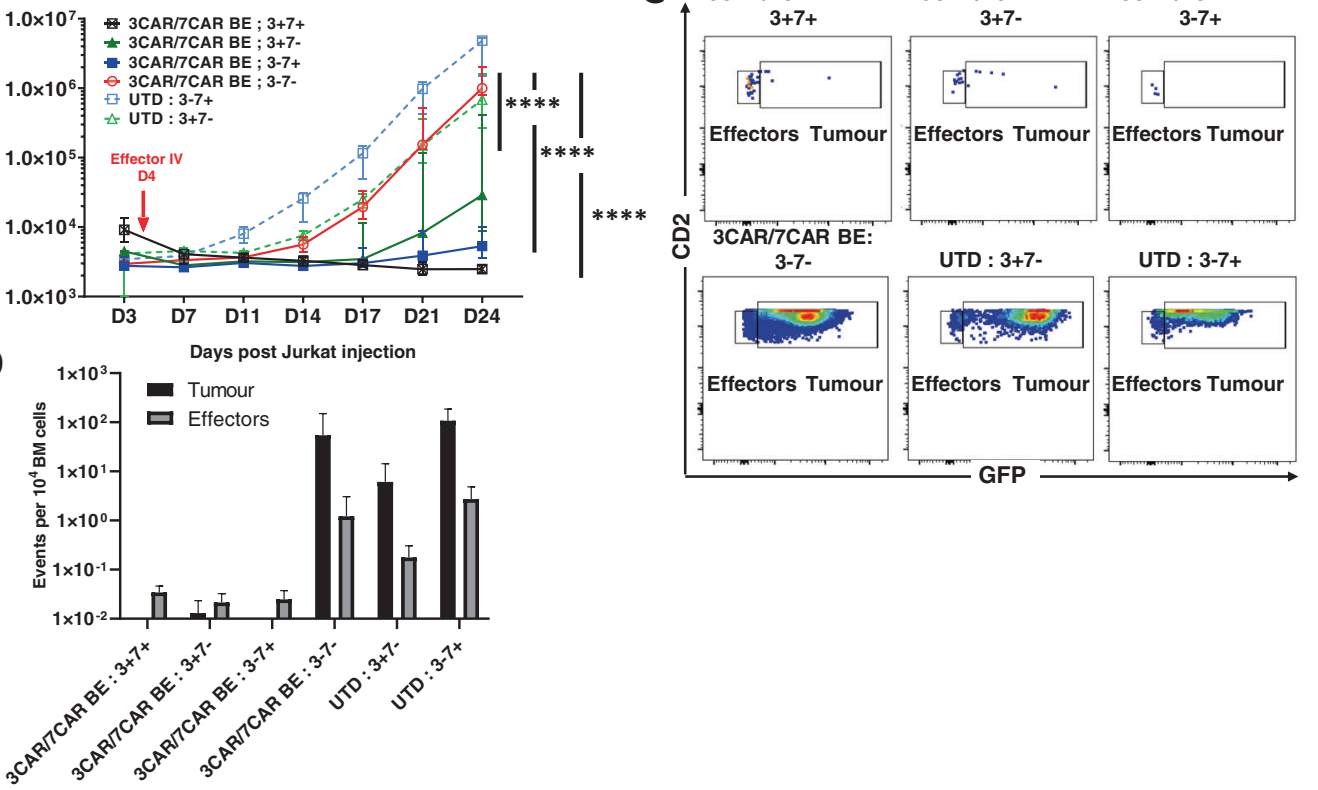

Fig. 6 3CAR and 7CAR cells effectively clear $T$ cell malignancy in vivo. A NSG mice were infused with $1 \times 10^{7} \mathrm{GFP}+\mathrm{LUC}+$ Jurkat $\mathrm{T}$ cells modified to express mixed $\mathrm{CD} 3$ and/or $\mathrm{CD} 7$ surface antigens in groups of $(n=5) \mathrm{CD}^{-} \mathrm{CD}^{-},(n=4) \mathrm{CD}^{+} \mathrm{CD}^{-},(\mathrm{n}=5) \mathrm{CD}^{-} \mathrm{CD}^{+}$ or $(n=5) \mathrm{CD}^{-} \mathrm{CD}^{-}$and imaged on day 3 prior to infusion of $1 \times 10^{7}$ $\mathrm{TCR}^{-} \mathrm{CD}^{-}$3CAR/7CAR mixed effectors or untransduced (UTD) cells. Leukaemic progression monitored by serial BLI for 24 days and revealed disease progression in animals receiving untransduced $\mathrm{T}$ cells $\left(3 \mathrm{CAR}^{-} 7 \mathrm{CAR}^{-}\right)$and in animals engrafted with antigen-negative $\left(\mathrm{CD} 3^{-} \mathrm{CD}^{-}\right)$leukaemia. B Bioluminescence signal of each animal plotted as Average radiance [photons $/ \mathrm{s} / \mathrm{cm}^{2} / \mathrm{sr}$ ]. Each line represents a

different experimental group and each point on the line the mean of each group. Error bars represent SEM. Area under the curve was calculated for each experimental group and values were compared using a one-way ANOVA with Tukey multiple comparison post-hoc $* * * P P<0.0001$. C Example of day 24 flow cytometry-based detection in bone marrow of $\mathrm{mCD} 11 \mathrm{~b}^{-} / \mathrm{hCD}^{2} 5^{+}$effector $\mathrm{T}$ cells $\left(\mathrm{CD} 2^{+} \mathrm{GFP}^{-}\right)$in $3 \mathrm{CAR} / 7 \mathrm{CAR}$ treated animals and residual leukaemia $\left(\mathrm{CD}^{+} \mathrm{GFP}^{+}\right)$in antigen-negative and untransduced groups. D Frequency of hCD $45^{+} \mathrm{CD} 2^{+} \mathrm{GFP}^{-}$effector events or $\mathrm{hCD} 5^{+} \mathrm{CD} 2^{+} \mathrm{GFP}^{+}$Jurkat events per $10^{4}$ acquired bone marrow events.

edited $\mathrm{T}$ cells compared to $5-8 \%$ for base edited cells (Supplementary Fig. 13C, D) in line with expectations.

To investigate the possibility of guide-dependent 'offtarget' effects of coBE3, the top 20 in silico predicted off-

target sites for both TRBC and CD7 targeting sequences were interrogated by deep sequencing for cytosine conversions. Levels of $\mathrm{C}>\mathrm{T}$ conversions in base edited $\mathrm{T}$ cells were $<1 \%$, at $19 / 20$ of the TRBC off-sites, and $1.55 \%$ at a 
single intronic site (TRBC-OT1, chr19) (Supplementary Fig. 13 and Supplementary Tables S1 and S2). For CD7, in $15 / 20$ predicted 'off target' sites, $\mathrm{C}>\mathrm{T}$ base conversions were broadly similar with frequencies $<1 \%$. Two sites were identified exhibiting up to $7.63 \%$ (CD7-OT1, chr17) and $4.08 \%$ (CD7-OT20, chr12) $\mathrm{C}>\mathrm{T}$ conversion within the 5 bp editing window, however, these were confirmed to be intronic and non-coding (Supplementary Fig. 13 and Supplementary Tables S1 and S2).

Finally, we investigated the possibility of promiscuous deamination effects on $\mathrm{scFv}$ antigen recognition elements that confer CAR antigen specificity. Somatic hypermutation of antibody variable regions mediated by human cytidine deaminase in $\mathrm{B}$ cells has long been recognised as a mechanism to increase receptor diversity [28]. Ectopic deamination BE effects in CAR-T cells could inadvertently redirect cells away from desired targets leading to unknown specificity. Thus, to interrogate the integrity of CAR coding sequences following editing, total RNA was extracted from SpCas9 and coBE3 mRNA modified $\mathrm{T}$ cells at 48 and $96 \mathrm{~h}$ post transduction and again on day 14, at end of 3CAR and 7CAR production. cDNA was synthesised using universal primers flanking the CAR sequences and analysed by NGS for cytidine-specific base conversion, focussing on the heavy and light antigen binding regions (ABRs) (Fig. 7). Quantification of cytidine transitions and transversions revealed that $\mathrm{C}->\mathrm{N}$ occurrences were infrequent, shared by SpCas9 and coBE3 edited samples, and generally ranged between $0 \%$ and $2 \%$ within the ABRs (Fig. 7A). Only two changes were detected above this level, and only transiently, after $48 \mathrm{~h}$ in 3CAR base edited $\mathrm{T}$ cells at positions P370 (4\%) and P718 (7\%) and had reduced at the end of production (Fig. 7). Importantly there was minimal C- $>\mathrm{T}$ transition across the entire CAR transgene in either SpCas9 or coBE3 modified cells and the frequency of events detected $48 \mathrm{~h}$ after mRNA delivery were broadly similar to those at the end of production on day 14 (Fig. 7B, C). Similar NGS interrogation of genomic DNA from end-ofproduction cells detected no permanent $\mathrm{C}>\mathrm{T}$ or $\mathrm{G}>\mathrm{A}$ conversions, and thus overall, there was no evidence of sustained corruption of antigen specificity in base edited CAR $\mathrm{T}$ cells.

\section{Discussion}

Engineered CAR T cell therapies for non-B cell malignancies are proving challenging, with particular difficulties arising when target antigens on target populations are also present on $\mathrm{T}$ cells. We have previously described strategies to address fratricide for the generation of CAR T cells against CD3, a definitive $\mathrm{T}$ cell marker present on certain $\mathrm{T}$ cell lymphomas and a proportion of T-ALL [9]. Electroporation of TALEN mRNA was used to prevent assembly and expression of the multimeric TCR $\alpha \beta / \mathrm{CD} 3$ complex. The resulting $\mathrm{T}$ cells were not only insensitive to the 3CAR but were simultaneously rendered TCR $\alpha \beta$ negative, and thus non-alloreactive and incapable of mediating graft versus host disease if derived from an unmatched healthy donor. Approaches using 'off-theshelf' universal CAR T cells against CD19 are already under investigation, including cells with additional genomic edits to address the risk of host-mediated rejection [29, 30]. CAR19 T cells edited at the TRAC and CD52 locus using TALEN technology are undergoing multicentre testing, and similar approaches using homing endonuclease editing or CRISPR/ Cas9 are under investigation [18-20, 23]. An ability to premanufacture universal CAR T cells opens up the possibility of generating cell banks with different specificities, which could then be used in combination as a strategy to address incomplete or internalised target expression and antigen escape. In the context of CAR T cells targeting $\mathrm{T}$ cell malignancies, a time-limited application is envisaged to allow recovery of a functional $\mathrm{T}$ cell compartment, derived either from autologous, antigen-negative precursors or donor-derived stem cells following allo-SCT. Suitable additional antigens for targeting T cell malignancies include TCR $\alpha \beta, \mathrm{CD} 2, \mathrm{CD} 5$ and CD7. A strategy to target TCR $\alpha \beta$ on $\mathrm{T}$ cell lymphomas is in trial and relies on discriminatory targeting of TRBC1 or TRBC2, but would not be suitable to combine with $\mathrm{CD} 3 \varepsilon$ given their linked multimeric expression [31]. CD2 is expressed on mature $\mathrm{T}$ cells and has a role in adhesion and costimulation upon interaction with its ligand CD58 (LFA-3) [32]. Alefacept, a recombinant LFA-3/IgG1 fusion protein was previously investigated as an immunosuppressive antiCD2 treatment for psoriasis, and was noted to selectively deplete memory $\mathrm{T}$ cell subsets $[33,34]$. In-depth functional studies of CD2 in animals are sparse, mainly because LFA-3 in mice is absent and normal lymphocyte compartments with CD2 knockouts may not be representative [35]. Monoclonal antibodies against CD5 have also been investigated and shown encouraging results against cutaneous T-cell lymphoma and T-ALL [36, 37]. The molecule is downregulated in activated T cells and anti-CD5 CAR T cells co-expressing a CD28 costimulatory domain exhibited only transient fratricide, but 4-1BB coding constructs exhibited impaired expansion of CD5 CAR T cells and higher levels of fratricide were attributed to tumour necrosis factor receptor-associated factor-mediated upregulation of intracellular adhesion molecule $1[38,39]$. We have focussed on $\mathrm{CD} 7$, which has previously been investigated as a target for monoclonal antibody immunotoxin therapy against $\mathrm{T}$ cell malignancies [40]. Recently the introduction of strategies to inhibit expression on $\mathrm{T}$ cells has enabled the anti-CD7 $\mathrm{scFv}$ binding domains to be employed in CAR approaches. Gomes-Silva et al reported combining CRISPR/Cas9 editing to eliminate CD7 in T cells expressing anti-CD7 CARs and have modelled strategies in humanised mice against $\mathrm{T}$ cell lymphoma and acute myeloid 


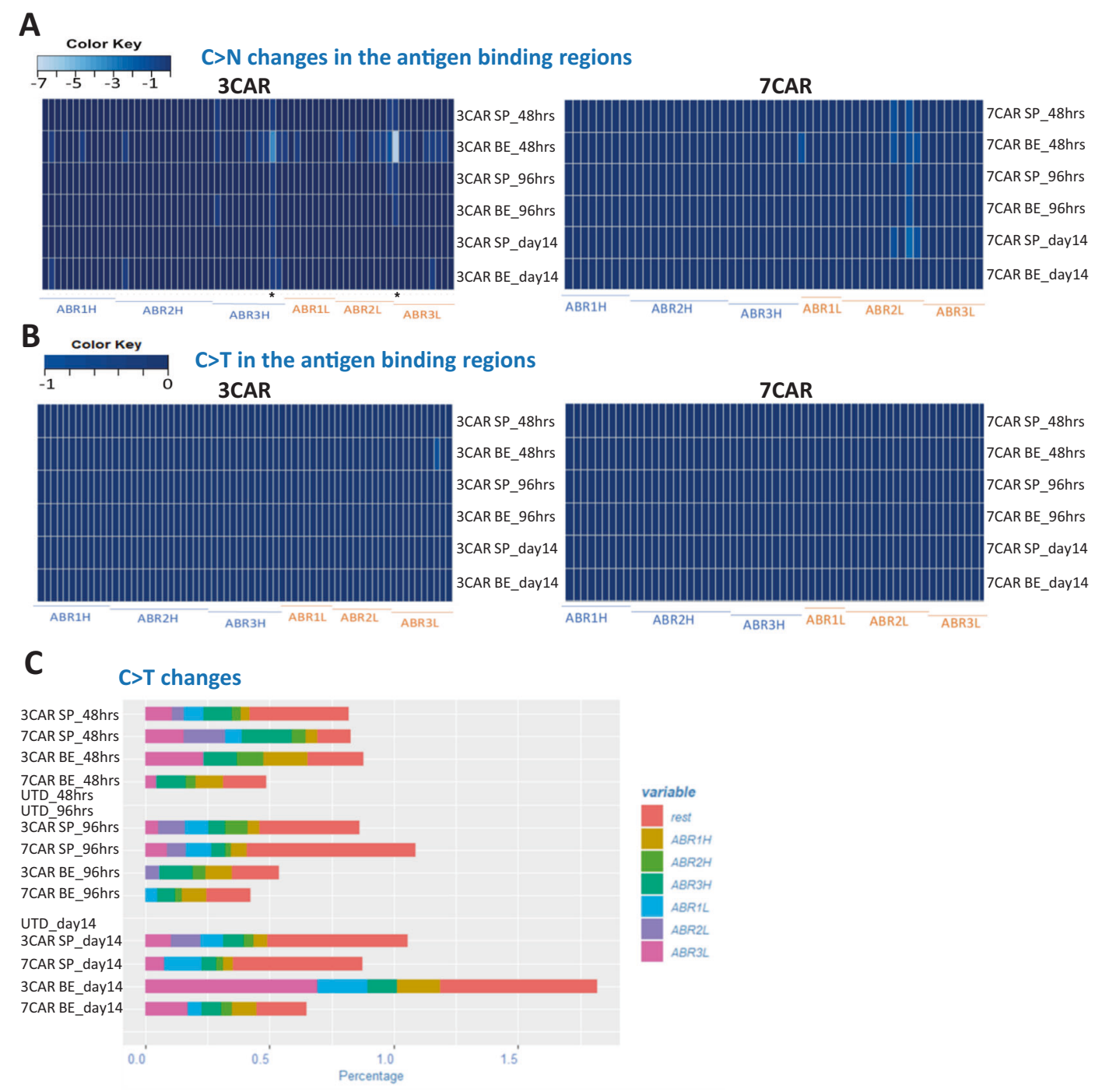

Fig. 7 Cytidine deamination does not compromise integrity of antigen specificity of CAR sequences. Serial examination of 3CAR or 7CAR scFv RNA sequences $48 \mathrm{~h}$ and $96 \mathrm{~h}$ after electroporation with SpCas9 (SP3/SP7) or coBE3 (BE3/BE7) mRNA and again at end of production on d14. A Amplicons of 3CAR (left) and 7CAR (right) $v H$ and $\mathrm{vL}$ sequences with antigen-binding regions (ABR) displayed mapped as a Heatmap in $\mathrm{R}$ using the gplots library for $\mathrm{C}>\mathrm{N}$ conversion rates at the marked sites. B 3CAR (left) and 7CAR (right) scFv ABR mapped as a Heatmap for $\mathrm{C}>\mathrm{T}$ conversion rates. $\mathrm{C}$ Stacked histogram showing $<2 \% \mathrm{C}>\mathrm{T}$ changes in each region $(\mathrm{ABR} 1 \mathrm{H}, \mathrm{ABR} 2 \mathrm{H}$, $\mathrm{ABR} 3 \mathrm{H}$ etc and outside the binding regions - ('rest') in SpCas-CAR3, SpCas-CAR7, BE-CAR3, BE-CAR7 or untransduced (UTD) samples. leukaemia [15]. In addition, an alternative approach using 'protein expression blockade' has employed an anti-CD7 $\mathrm{scFv}$ moiety coupled to endoplasmic reticulum/Golgi retention elements to anchor the protein and prevent surface expression [13]. Of note, these recent reports have found no evidence of impaired effector cytotoxicity after disruption of CD7, and CD7 deficient mice appear to retain normal lymphoid compartments [41]. The cytoplasmic tail of CD7 activates phosphoinositol signalling of CD7 in T cells and may play a role in co-stimulation [42]. Secreted epithelial protein K12/SECTM1 is the ligand for CD7 and is an interferoninducible protein encoded at the continuous genetic locus for CD7, indicating feedback through transcriptional regulation may be in operation [43, 44]. Given that CAR T cells disrupted for $\mathrm{CD} 7$ retain potent functional activity, it appears that CD7 signalling, and by proxy SECTM1 activity, may be redundant for anti-tumour function.

In consideration of allogeneic cells, Cooper et al. described a strategy to generate 'universal' donor T cells with simultaneous TCR/CD3 and CD7 disruption using CRISPR/Cas9 editing to generate cells expressing their anti-CD7 CAR [14]. The cells were investigated against $\mathrm{T}$ cell lines and primary $\mathrm{T}$ cell malignancies, and included studies in chimeric xenografted mice. The issue of possible translocations from multiplexed editing was not investigated, but we report a similar editing frequency and found readily detectable and 
quantifiable events between sites of guide activity at the $T R B C$ and $C D 7$ loci. The frequency was consistent with past experiences with TALENs and CRISPR/Cas9. In contrast, upon switching to BE3, and using the same sgRNA guides, these events were eliminated.

Given that additional genome edits would be required to confer resistance to Alemtuzumab or disrupt expression of checkpoint pathways such as PD1, the frequency of additional translocation events would become problematic. We have found that additive co-delivery of sgRNAs against CD52 conferred high levels of triple knockout (TCR $\alpha \beta /$ CD7/CD52) with no alternation in phenotype or cytotoxic responses (data not shown) and anticipate transformation risks will be mitigated compared to conventional Cas9. However, definitive experiments to prove an enhanced safety profile in $\mathrm{T}$ cells are difficult to conceive given that mature $\mathrm{T}$ cells are not readily transformed. Similarly, it is not readily possible to quantify adverse impacts that may arise from ectopic deamination activity, either as a result of guide-directed BE effects at possible off-target sites, or as a result of promiscuous, guide-independent, APOBEC activity on DNA or RNA. Interrogation of 20 in silico predicted guide- dependent Cas9 specific 'off-targets' for each target site found only infrequent and low level ectopic activity. One potentially serious consequence of promiscuous base conversion would be changed to antigen receptor coding sequences, in particular if involving regions defining antigen-binding specificity. Interrogation of RNA collected at serial timepoints over a 14 day period from electroporation of BE mRNA until end of production found no evidence of deamination mediated transition, transversion (or other aberrations) within the antigen-binding frameworks of the $\mathrm{scFv}$ for 3CAR or 7CAR at the end of production. This finding is reassuring, but in any case, unwanted activity has been greatly reduced in recent $\mathrm{BE}$ variants.

The processes described here are readily scalable and similar techniques have already been deployed in a compliant manner for CRISPR/Cas9 edited CAR19 T cells [23]. The issue of stringently depleting residual TCR $\alpha \beta$ T cells remains critical for non-HLA matched donor $\mathrm{T}$ cells, and the CliniMACS Prodigy device supports automated microbead depletion with $<1 \%$ TCR $\alpha \beta$ expression in the final products. This step is retained for anti-T cell CARs even though CAR-mediated 'self-enrichment' arises during production and will ensure strict specification limits. It is anticipated that sufficient production for both 3CAR and 7CAR will be achievable from a single non-mobilised peripheral blood leukapheresis to support dozens of therapeutic doses, offering a route to affordable and accessible cell therapy. Early therapeutic applications are anticipated against relapsed refractory T-ALL in combination with allogeneic SCT.

\section{Materials and methods}

\section{Cell lines}

Jurkat cells (acute T cell leukaemic cell line from ATCC) were modified to express $\mathrm{CD}^{+} \mathrm{CD}^{+} \mathrm{CD}^{+} \mathrm{CD} 7^{-}$, $\mathrm{CD}^{-} \mathrm{CD}^{+}$and $\mathrm{CD}^{-}{ }^{-} \mathrm{CD} 7^{-}$by $\mathrm{SpCas} 9$ mRNA-based disruption of TCR and/or CD7 using TRBC sgRNA and CD7 sgRNA and maintained in culture in RPMI-1640 (Thermo Fisher Scientific) supplemented with 10\% FBS (Millipore Sigma) (Supplementary Fig. 9). For in vivo experiments cells were stably transduced with a 3rd generation pCCL-PGK-EGFP-LUC lentiviral vector and were sorted on a MoFlo XDP (BD) for GFP expression prior to banking.

\section{SpCas9 and coBE3 genome editing}

Synthetic sgRNAs were manufactured by Synthego (California, US) by automated solid-phase synthesis with $2^{\prime}$-Omethyl $3^{\prime}$ phosphorothioate modifications. Single guide RNA containing a 20 nucleotide protospacer with an 80 nucleotide CRISPR scaffold were eluted in nuclease-free Tris-EDTA buffer.

sgRNA guide sequences were selected for compatibility with both SpCas9 and coBE3: TRBC 1 and 2/Exon 15' CC CACCAGCTCAGCTCCACG 3'; CD7 Exon 25' CAC CTGCCAGGCCATCACGG $3^{\prime}$.

$\mathrm{BE}$ guides were designed to mediate cytidine to uracil to thymidine $(\mathrm{C}>\mathrm{U}>\mathrm{T})$ modification in exon 1 of $\mathrm{TRBC} 1 / 2$ with an intended amino acid conversion of Tryptophan (Trp) to stop (STOP*) (protospacer positions G > A and/or $\mathrm{G}_{6}>\mathrm{A}$ ). CD7 exon 2-targeting sgRNA promoted the deamination-mediated conversion of Glutamine (Gln) to a premature stop (STOP*) (protospacer positions $\mathrm{C}_{8}>\mathrm{T}$ ).

CleanCap ${ }^{\circ}$ Cas 9 mRNA encoded SpCas9 was supplied by Trilink US. Custom-made codon optimised BE3 (coBE3) was supplied as CleanCap (Trilink US) with a Cap 1 structure, and polyadenylated to increase expression and stability.

\section{Generation of 3CAR and 7CAR vectors}

3CAR expressing OKT3 monoclonal antibody scFv was previously described [9]. 7CAR was derived by codon optimisation (GeneArt) of variable heavy chain and variable light chain antigen-binding elements of the anti-human CD7 murine hybridoma, 3A1e sequence (GenBank: AAA83268.1) [15]. The scFv was fused to a CD8 transmembrane domain and to activation domains derived from $41 \mathrm{BB}$ and $\mathrm{CD} 3 \zeta$. The resultant 7CAR construct was cloned into a lentiviral vector ( $\mathrm{pCCL}$ ) backbone under the control of a PGK promoter. Vector stocks pseudotyped with a vesicular stomatitis virus glycoprotein (VSV-G) envelope 
were generated in $293 \mathrm{~T}$ cells (ATCC) and yielded titres $>5 \times 10^{8}$ transducing units $/ \mathrm{ml}$ after ultracentrifugation [45].

\section{Generation of fratricide resistant 3CAR and 7CAR effectors}

PBMCs were obtained from consented healthy donors (human sample collections were approved by University College London (UCL) and Anthony Nolan ethics committees and collected with written consent), and were cultured in 48-well plates at a density of $1 \times 10^{6} / \mathrm{ml}$ in TexMACS (Miltenyi Biotec), $3 \%$ human serum (Seralab) $+20 \mathrm{ng} / \mathrm{ml}$ human recombinant IL-2 (Miltenyi Biotec) and activated with TransAct reagent (Miltenyi Biotec). Activated T cells were electroporated with $20 \mu \mathrm{g} / \mathrm{ml}$ TRBC sgRNA (Synthego), $20 \mu \mathrm{g} / \mathrm{ml}$ CD7 sgRNA (Synthego) and $50 \mu \mathrm{g} / \mathrm{ml}$ codon optimised BE3 mRNA (TriLink Biotechnologies) in a Lonza 4D or LV nucleofector (Lonza, Slough, UK) and cultured at $30^{\circ} \mathrm{C} 5 \%$ $\mathrm{CO}_{2}$ overnight before returning to $37^{\circ} \mathrm{C}$. Cells were transduced with 3CAR or 7CAR lentiviral vector preparations at an MOI of 5 the following day. Cells were cultured in G-Rex 24 well, G-Rex 10 or G-Rex 100 chambers as per manufacturer's instructions (Wilson Wolf, MN, USA).

\section{Flow cytometry}

Cells were stained with the following primary anti-human antibodies: mouse anti-human CD2 (clone LT2), mouse antihuman CD3 (clone BW264/56), mouse anti-human TCR $\alpha \beta$ (clone BW242/412), mouse anti-human CD4 (clone VIT4), mouse anti-human CD8 (clone BW135/80), mouse antihuman CD45RA (T6D11), mouse anti-human CD62L (145/ 15), mouse anti-human CD19 (clone LT19), mouse antihuman CD56 (clone AF12-7H3), (all from Miltenyi Biotec), mouse anti-human CD7 (clone M-T701) (BD), mouse antihuman $\mathrm{CD} 7$ (clone 6B7) (BioLegend), mouse anti-human PD-1 (EH12.1) (BD), mouse anti-human TIM-3 (7D3) (BD), mouse anti-human LAG-3 (T47-530) (BD). To assess the efficiency of 3CAR and 7CAR transduction, cells were stained using a Biotin-SP (long spacer) AffiniPure Fab Fragment Goat Anti-Mouse IgG fragment-specific antibody (catalogue 115-066-072; Jackson Immunoresearch, Stratech Scientific Limited) followed by Streptavidin-APC (catalogue 405207, BioLegend) or Streptavidin-FITC (catalogue 405202, BioLegend). Cells were acquired on a Cyan or on a BD LSRII (BD Biosciences), and analysis was performed using FlowJo v10 (TreeStar Inc.).

\section{Molecular quantification of on-target genome editing}

Genomic DNA extraction was performed using DNeasy Blood and Tissue Kit (69504, QIAGEN) and a PCR reaction designed to amplify $400-800 \mathrm{bp}$ over the protospacer binding site.
Primers used to amplify genomic loci for TRBC were TRBC FWD: 5' ACACAGAGCCCCTACCAG 3'; TRBC REV: $5^{\prime}$ GCTACCTGGATCTTTCCA $3^{\prime}$ and for CD7 were CD7 FWD: 5' ATCACCTGCTCCACCAGCGG 3'; CD7 REV 5' GTGTGTGCTGGCGAGGACAC $3^{\prime}$. PCR products were gel extracted and sent for Sanger sequencing (Eurofins Genomics). Resulting Sanger sequencing data was analysed using TIDE (https://tide.nki.nl/), to measure the frequency of indels, at the predicted SpCas9 scission site (Supplementary Figs. 1B and 2B). When analysing $\mathrm{C}>\mathrm{T}$ conversion rates produced by coBE3, EDITR software was used (https://moriaritylab.shinya pps.io/editr_v10/).

\section{Translocation detection and quantification}

To detect potential chromosomal translocations following multiplexed editing, primers flanking genome editing region at TRBC locus within chromosome $7 \mathrm{q}$-arm and CD7 locus within chromosome 17 q-arm were designed amplifying translocations T1-T4. TRBC Fwd - CD7 Fwd (T1), TRBC Rev - CD7 Fwd (T2), TRBC Rev-CD7 Rev (T3) and TRBC Fwd - CD7 Rev (T4) primer combinations were used to PCR amplify SpCas9 or coBE3 edited mixed 3CAR/7CAR or untransduced DNA products. Plasmids carrying the predicted fusions were synthesised as positive controls (GeneArt).

A ddPCR (BioRad) was used for the accurate quantification of the TRBC:CD7 translocations. Each sample was run as a duplexed assay consisting of translocation-specific primer pairs (designed for each translocation for a maximum of $230 \mathrm{bp}$ product) and probes as well as an internal (albumin) reference primer and probe set (Supplementary Table S3). All primers and probes were ordered from IDT. PCR reactions were set up using the ddPCR Supermix for Probes, no dUTP (BioRad). Droplets were generated and analysed using the automated QX200 AutoDG Droplet Digital PCR System by BioRad. Analysis was done on Quantasoft (BioRad) and in R using an optimised version of twoddPCR (https://github.com/CRUKMI-Computationa lBiology/twoddpcr/) for the purpose of this study.

\section{Molecular characterisation of on and off-target DNA editing}

Online software Benchling was used to predict off-targets for TRBC and CD7 guide sequences. NGS libraries were prepared for the top 20 'off-target' sites using a Nextera XT Kit (Illumina, Cambridge, UK). Products were amplified using combinations of target-specific primers (Supplementary Tables S1, S2 and S4). After the library preparation, individually barcoded samples were pooled and run in MiSeq using a 500-V2 nano-cartridge. Demultiplexed fastq files were uploaded to Galaxy [46] for quality check, trimming and alignment. Base conversions and NHEJ signatures were 
analysed using Naïve Variant Caller and Pindel, respectively [47]. Figures were created in R.

\section{Detection of Cas9 protein}

Cell pellets $\left(1 \times 10^{6}\right.$ cells $)$ were acquired at $12,24,48,72 \mathrm{~h}$ and 7 days post electroporation with coBE3 mRNA $(50 \mu \mathrm{g} / \mathrm{mL})$. Total protein was quantified by a bicinchoninic acid (BCA) assay. Western blot was run using $25 \mu \mathrm{g}$ total protein per sample. $16.5 \mathrm{ng}$ Alt-R ${ }^{\circledR}$ S.p. HiFi Cas9 Nuclease V3 (1081061, IDT, Leuven, Belgium) was used as positive control. Membrane was blotted with mouse anti-CRISPRCas9 antibody (ab191468, abcam, Cambridge, UK) at a 1:1000 dilution in $3 \%$ milk overnight at $4{ }^{\circ} \mathrm{C}$ before incubation with secondary HRP-linked sheep anti mouse (NA931-1ML, GE Healthcare Life Sciences, Buckinghamshire, UK) at a 1:3000 dilution in $5 \%$ milk for $1 \mathrm{~h}$ at room temperature. Protein was visualised by chemiluminescence using Pierce ECL western blotting substrate (32106, ThermoFisher Scientific).

\section{Chromium release assay of in vitro cytotoxicity}

Cytotoxic activity of 3CAR and 7CAR cells was assessed by ${ }^{51} \mathrm{Cr}$ release assay. For this, $5 \times 10^{351} \mathrm{Cr}$-labelled $\mathrm{CD} 3^{+}$ $\mathrm{CD}^{+}, \mathrm{CD}^{+} \mathrm{CD}^{-}, \mathrm{CD}^{-} \mathrm{CD}^{+}, \mathrm{CD}^{-}{ }^{-} \mathrm{CD} 7^{-}$or a $1: 1 \mathrm{mix}$ of $\mathrm{CD}^{+} \mathrm{CD}^{-}: \mathrm{CD}^{-} \mathrm{CD}^{+}$Jurkat cells, were incubated with either 3CAR, 7CAR, mixed 3CAR/7CAR or untransduced control effector cells at increasing effector:target ratios (E:T ratios) in 96-well microplates for $4 \mathrm{~h}$ at $37^{\circ} \mathrm{C}$. Supernatant was subsequently harvested and mixed with OptiPhase HiSafe 3 (PerkinElmer) scintillation fluid and incubated at RT for $16 \mathrm{~h} .{ }^{51} \mathrm{Cr}$ release was then measured in a microplate scintillation counter (Wallac 1450 MicroBeta TriLux). Specific lysis was calculated using the formula [(experimental release-spontaneous release)/(maximum release - spontaneous release) $\times 100]$.

\section{In vivo antitumor activity}

Ten-week-old female NOD/SCID $/ \mathrm{\gamma c}^{-1-}$ NSG mice (Charles River, strain: NSG [005557] from The Jackson Laboratory), were inoculated i.v. with $1 \times 10^{7} \mathrm{CD}^{+} \mathrm{CD}^{+}, \mathrm{CD}^{+} \mathrm{CD}^{-}$, $\mathrm{CD}^{-}{ }^{-} \mathrm{CD}^{+}$and $\mathrm{CD}^{-}{ }^{-} \mathrm{CD} 7^{-}$Jurkat $\mathrm{T}$ cell tumour targets by tail vein injection on day 0 . The Jurkat $\mathrm{T}$ cell targets had been stably transduced to express $\mathrm{EGFP}^{+} \mathrm{LUC}^{+}$and, following CRISPR/Cas9-mediated TRBC1/2 and/or CD7 disruption, had been sorted for $\mathrm{CD}^{+}$and $\mathrm{CD}^{-}, \mathrm{CD}^{+}$ or $\mathrm{CD}^{-}$expressers. Tumour engraftment was confirmed by in vivo imaging of bioluminescence using an IVIS Lumina III In vivo Imaging System (PerkinElmer, live image version 4.5.18147) on day 3 and mice were further injected on day 4 with either PBS, $1 \times 10^{7}$ untransduced T cells, $10 \times 10^{7} 3$ CAR SP T cells, $1 \times 10^{7} 3$ CAR BE T cells, $1 \times 10^{7} 7$ CAR SP
T cells, $1 \times 10^{7} 7$ CAR BE T cells or $1 \times 10^{7}$ mixed 3CAR/ 7CAR T cells. Analysis of tumour clearance was performed by serial bioluminescent imaging on days $3,10,17,24$ and 31 (Fig. 5A and Supplementary Figs. 10 and 11), or days 3, 7, 11, 14, 17, 21 and 24 (Fig. 6) and processing of BM for the monitoring of tumour progression vs. clearance was carried out on day 24 and 31. BM samples were processed by a RBC lysis, followed by staining for flow cytometry. All animal studies were approved by the UCL Biological Services Ethical Review Committee and licensed under the Animals (Scientific Procedures) Act 1986 (Home Office, London, United Kingdom).

\section{Primary T-ALL blasts}

Primary T-ALL patient samples were provided by the Bloodwise Childhood Leukemia Cell Bank, Stockport, United Kingdom. To phenotype, patient cells were stained with the following primary antibodies: mouse anti-human CD3 (clone BW264/56; Miltenyi Biotec), mouse antihuman CD7 (clone M-T701) (BD Biosciences).

For the FACS-based killing assay, $10^{5}$ T-ALL patient target cells loaded with CFSE dye (CellTrace, Invitrogen) were incubated for $24 \mathrm{~h}$ at 1:1 ratio with either 3CAR, 7CAR, mixed 3CAR/7CAR effector T cells or control untransduced T cells.

Study approval. Human sample collections were approved by University College London (UCL) ethics committee and collected with written consent. Leukaemia samples were provided by the Bloodwise Childhood Leukemia Cell Bank.

\section{CAR and 7CAR targeted RNA sequencing}

$\mathrm{T}$ cells were treated with either SpCas9 or coBE3 then transduced to express 3CAR or 7CAR. RNA was extracted from samples at different timepoints using the RNeasy mini kit (Qiagen). DNase treatment was performed with RQ1 DNase (Promega) followed by reverse transcription using a primer specific to both the 3 and 7 CAR maps (CAGGGCCTGCATGTGCAG). The resulting cDNA was then purified using the Qiagen PCR purification kit and amplified using primers specific to 3 and 7 CAR sequences (Fwd: GGCGGAGGATCTGGCGGA and Rev: GACGT GTACGTCCGGGAC). PCR products were size-selected using Promega beads. Once quantified by Qubit fluorometry and checked using Tapestation electrophoresis, libraries were prepared for sequencing following the Illumina Nextera XT protocols. Barcoded libraries were pooled, denatured and finally, sequenced on a MiSeq with a 500-V2 cartridge. Fastq files were downloaded using basespace Illumina and analysed with a homebrewed pipeline including steps for quality check (fastp), trimming (TrimGalore), removing PCR duplicates (Mark Duplicate reads), 
alignment to reference(s) (Bowtie2) but also investigation for minor allele frequencies (Naïve Variant Caller) and indels (Pindel). Sequences were visualised on Integrative Genomics Viewer. All figures were produced in Rstudio. Genomic DNA was similarly sequenced using primers flanking the antigen-specific binding sequences of 3CAR and CAR7 (Fwd: CTGCTGCTGTGGGTGCTGCT and Rev: CAGGGCCTGCATGTGCAG).

\section{Statistics}

For comparison of in vitro generated data, values are presented as mean percentages with SEM. A Mann-Whitney statistical test has been performed when $n=4$ samples from each group are compared. For comparison of in vivo study groups, area under the curve was calculated for the grouped data. Values were compared using a one-way ANOVA with Tukey multiple comparison post-hoc. Values from 3 or more samples are presented as median with interquartile range or as mean with SEM. In all experiments, $P<0.05$ was considered statistically significant. All statistical analysis was performed using GraphPad Prism software version 8.0.

\section{Disclosures}

WQ holds interests unrelated to this project in Autolus Ltd. WQ received unrelated research funding from Cellectis, Servier, Miltenyi, Bellicum. UCL Business has filed patents in relation to the generation of genome-edited CAR T cells. This work is (partly) funded by the NIHR GOSH BRC. The views expressed are those of the author(s) and not necessarily those of the NHS, the NIHR or the Department of Health.

Acknowledgements We would like to thank Ailsa Greppi and Kyle O'Sullivan for their invaluable technical support with in vivo studies. Dr. Ayad Eddaudi at the UCL Joint Great Ormond Street Institute of Child Health and Institute of Ophthalmology Flow Cytometry Core Facility, supported by the Great Ormond Street Children's Charity (GOSHCC), grant reference U09822 (October 2007), UCL Capital Equipment Funding, School of Life and Medical Sciences (September 2012), and UK Research and Innovation, grant reference MR/ L012758/1 (March 2014). The Anthony Nolan Trust (803716/ SC038827) for their support and provision of healthy blood donations. Synthego for the Genome Engineering Innovation Grant facilitating the procurement of sgRNAs. This work is (partly) funded by the NIHR GOSH BRC.

Funding Supported by the Wellcome Trust (215619/Z/19/Z), NIHR (RP-2014-05-007), NIHR Blood and Transplant Research Units (BTRU) and Great Ormond Street Biomedical Research Centre (ISBRC-1215-20012) Bloodwise (18011) and Children with Cancer (2014/171).

\section{Compliance with ethical standards}

Conflict of interest The authors declare no competing interests.
Publisher's note Springer Nature remains neutral with regard to jurisdictional claims in published maps and institutional affiliations.

Open Access This article is licensed under a Creative Commons Attribution 4.0 International License, which permits use, sharing, adaptation, distribution and reproduction in any medium or format, as long as you give appropriate credit to the original author(s) and the source, provide a link to the Creative Commons license, and indicate if changes were made. The images or other third party material in this article are included in the article's Creative Commons license, unless indicated otherwise in a credit line to the material. If material is not included in the article's Creative Commons license and your intended use is not permitted by statutory regulation or exceeds the permitted use, you will need to obtain permission directly from the copyright holder. To view a copy of this license, visit http://creativecommons. org/licenses/by/4.0/.

\section{References}

1. Litzow MR, Ferrando AA. How I treat T-cell acute lymphoblastic leukemia in adults. Blood. 2015;126:833-41.

2. Kadia TM, Gandhi V. Nelarabine in the treatment of pediatric and adult patients with T-cell acute lymphoblastic leukemia and lymphoma. Expert Rev Hematol. 2017;10:1-8.

3. Brammer JE, Saliba RM, Jorgensen JL, Ledesma C, Gaballa S, Poon M, et al. Multi-center analysis of the effect of T-cell acute lymphoblastic leukemia subtype and minimal residual disease on allogeneic stem cell transplantation outcomes. Bone Marrow Transpl. 2017;52:20-7.

4. Maude SL, Laetsch TW, Buechner J, Rives S, Boyer M, Bittencourt $\mathrm{H}$, et al. Tisagenlecleucel in children and young adults with B-cell lymphoblastic leukemia. N Engl J Med. 2018;378:439-48.

5. Park JH, Riviere I, Gonen M, Wang X, Senechal B, Curran KJ, et al. Long-Term Follow-up of CD19 CAR Therapy in Acute Lymphoblastic Leukemia. N Engl J Med. 2018;378:449-59.

6. Schuster SJ, Svoboda J, Dwivedy Nasta S, Porter DL, Chong EA, Mahnke Y, et al. Phase IIa trial of chimeric antigen receptor modified $\mathrm{T}$ cells directed against CD19 (CTL019) in patients with relapsed or refractory CD19+ lymphomas. Blood. 2014;124:3087-.

7. Bhoj VG, Arhontoulis D, Wertheim G, Capobianchi J, Callahan CA, Ellebrecht CT, et al. Persistence of long-lived plasma cells and humoral immunity in individuals responding to CD19directed CAR T-cell therapy. Blood. 2016;128:360-70.

8. Porter DL, Hwang WT, Frey NV, Lacey SF, Shaw PA, Loren $\mathrm{AW}$, et al. Chimeric antigen receptor $\mathrm{T}$ cells persist and induce sustained remissions in relapsed refractory chronic lymphocytic leukemia. Sci Transl Med. 2015;7:303ra139.

9. Rasaiyaah J, Georgiadis C, Preece R, Mock U, Qasim W. TCR alphabeta/CD3 disruption enables CD3-specific antileukemic $\mathrm{T}$ cell immunotherapy. JCI Insight. 2018;3.

10. Fry TJ, Shah NN, Orentas RJ, Stetler-Stevenson M, Yuan CM, Ramakrishna S, et al. CD22-targeted CAR T cells induce remission in B-ALL that is naive or resistant to CD19-targeted CAR immunotherapy. Nat Med. 2018;24:20-8.

11. Ruella M, Barrett DM, Kenderian SS, Shestova O, Hofmann TJ, Perazzelli J, et al. Dual CD19 and CD123 targeting prevents antigen-loss relapses after CD19-directed immunotherapies. J Clin Invest. 2016;126:3814-26.

12. Jia H, Wang Z, Wang Y, Liu Y, Dai H, Tong C, et al. Haploidentical CD19/CD22 bispecific CAR-T cells induced MRDnegative remission in a patient with relapsed and refractory adult B-ALL after haploidentical hematopoietic stem cell transplantation. J Hematol Oncol. 2019;12:57.

13. Png YT, Vinanica N, Kamiya T, Shimasaki N, Coustan-Smith E, Campana D. Blockade of CD7 expression in T cells for effective 
chimeric antigen receptor targeting of T-cell malignancies. Blood Adv. 2017;1:2348-60.

14. Cooper ML, Choi J, Staser K, Ritchey JK, Devenport JM, Eckardt $\mathrm{K}$, et al. An "off-the-shelf" fratricide-resistant CAR-T for the treatment of $\mathrm{T}$ cell hematologic malignancies. Leukemia. 2018;32:1970-83.

15. Gomes-Silva D, Srinivasan M, Sharma S, Lee CM, Wagner DL, Davis TH, et al. CD7-edited T cells expressing a CD7-specific CAR for the therapy of T-cell malignancies. Blood. 2017;130:285-96.

16. Li S, Wang X, Yuan Z, Liu L, Luo L, Li Y, et al. Eradication of TALL cells by CD7 targeted universal CAR-T cells and initial test of ruxolitinib-based CRS management. Clin Cancer Res. 2020.

17. Komor AC, Kim YB, Packer MS, Zuris JA, Liu DR. Programmable editing of a target base in genomic DNA without doublestranded DNA cleavage. Nature. 2016;533:420-4.

18. Poirot L, Philip B, Schiffer-Mannioui C, Le Clerre D, ChionSotinel I, Derniame S, et al. Multiplex genome-edited T-cell manufacturing platform for "off-the-shelf" Adoptive T-cell immunotherapies. Cancer Res. 2015;75:3853-64.

19. Qasim W, Zhan H, Samarasinghe S, Adams S, Amrolia P, Stafford $\mathrm{S}$, et al. Molecular remission of infant B-ALL after infusion of universal TALEN gene-edited CAR T cells. Sci Transl Med. 2017;9.

20. Benjamin R, Graham C, Yallop D, Jozwik A, Mirci-Danicar OC, Lucchini $\mathrm{G}$, et al. Genome-edited, donor-derived allogeneic antiCD19 chimeric antigen receptor T cells in paediatric and adult Bcell acute lymphoblastic leukaemia: results of two phase 1 studies. Lancet. 2020;396:1885-94.

21. Stadtmauer EA, Fraietta JA, Davis MM, Cohen AD, Weber KL, Lancaster E, et al. CRISPR-engineered $\mathrm{T}$ cells in patients with refractory cancer. Science. 2020;367.

22. Webber BR, Lonetree CL, Kluesner MG, Johnson MJ, Pomeroy EJ, Diers MD, et al. Highly efficient multiplex human $\mathrm{T}$ cell engineering without double-strand breaks using Cas9 base editors. Nat Commun. 2019;10:5222.

23. Georgiadis C, Preece R, Nickolay L, Etuk A, Petrova A, Ladon D, et al. Long terminal repeat CRISPR-CAR-coupled "Universal" $\mathrm{T}$ cells mediate potent anti-leukemic effects. Mol Ther. 2018;26:1215-27.

24. Preece R, Pavesi A, Gkazi SA, Stegmann KA, Georgiadis C, Tan ZM, et al. CRISPR-mediated base conversion allows discriminatory depletion of endogenous $\mathrm{T}$ cell receptors for enhanced synthetic immunity. Mol Ther Methods Clin Dev. 2020;19:149-61.

25. Grunewald J, Zhou R, Garcia SP, Iyer S, Lareau CA, Aryee MJ, et al. Transcriptome-wide off-target RNA editing induced by CRISPR-guided DNA base editors. Nature. 2019;;569:433-7.

26. Grunewald J, Zhou R, Iyer S, Lareau CA, Garcia SP, Aryee MJ, et al. CRISPR DNA base editors with reduced RNA off-target and self-editing activities. Nat Biotechnol. 2019;37:1041-8.

27. Zhou C, Sun Y, Yan R, Liu Y, Zuo E, Gu C, et al. Off-target RNA mutation induced by DNA base editing and its elimination by mutagenesis. Nature. 2019;571:275-8.

28. Maul RW, Gearhart PJ. AID and somatic hypermutation. Adv Immunol. 2010;105:159-91.

29. Ren J, Liu X, Fang C, Jiang S, June CH, Zhao Y. Multiplex genome editing to generate universal CAR T cells resistant to PD1 inhibition. Clin Cancer Res. 2017;23:2255-66.

30. Ren J, Zhang X, Liu X, Fang C, Jiang S, June CH, et al. A versatile system for rapid multiplex genome-edited CAR $\mathrm{T}$ cell generation. Oncotarget. 2017;8:17002-11.

31. Maciocia PM, Wawrzyniecka PA, Philip B, Ricciardelli I, Akarca $\mathrm{AU}$, Onuoha SC, et al. Targeting the T cell receptor beta-chain constant region for immunotherapy of $\mathrm{T}$ cell malignancies. Nat Med. 2017;23:1416-23.
32. Miller GT, Hochman PS, Meier W, Tizard R, Bixler SA, Rosa $\mathrm{MD}$, et al. Specific interaction of lymphocyte function-associated antigen 3 with $\mathrm{CD} 2$ can inhibit $\mathrm{T}$ cell responses. J Exp Med. 1993;178:211-22.

33. Chamian F, Lin SL, Lee E, Kikuchi T, Gilleaudeau P, SullivanWhalen $\mathrm{M}$, et al. Alefacept (anti-CD2) causes a selective reduction in circulating effector memory $\mathrm{T}$ cells $(\mathrm{Tem})$ and relative preservation of central memory $\mathrm{T}$ cells $(\mathrm{Tcm})$ in psoriasis. J Transl Med. 2007;5:27.

34. Ellis CN, Krueger GG, Alefacept Clinical Study G. Treatment of chronic plaque psoriasis by selective targeting of memory effector T lymphocytes. N Engl J Med. 2001;345:248-55.

35. da Silva AJ, Brickelmaier M, Majeau GR, Li Z, Su L, Hsu YM, et al. Alefacept, an immunomodulatory recombinant LFA-3/IgG1 fusion protein, induces CD16 signaling and CD2/CD16-dependent apoptosis of $\mathrm{CD} 2(+)$ cells. J Immunol. 2002;168: 4462-71.

36. Feng J, Xu H, Cinquina A, Wu Z, Chen Q, Zhang $\mathrm{P}$, et al. Treatment of aggressive t cell lymphoblastic lymphoma/leukemia using anti-CD5 CAR T cells. Stem Cell Rev Rep. 2021.

37. Klitgaard JL, Koefoed K, Geisler C, Gadeberg OV, Frank DA, Petersen J, et al. Combination of two anti-CD5 monoclonal antibodies synergistically induces complement-dependent cytotoxicity of chronic lymphocytic leukaemia cells. $\mathrm{Br} \mathrm{J}$ Haematol. 2013;163:182-93.

38. Mamonkin M, Mukherjee M, Srinivasan M, Sharma S, GomesSilva D, Mo F, et al. Reversible transgene expression reduces fratricide and permits 4-1BB costimulation of CAR $\mathrm{T}$ cells directed to T-cell malignancies. Cancer Immunol Res. 2018;6: $47-58$.

39. Mamonkin M, Rouce RH, Tashiro H, Brenner MK. A T-celldirected chimeric antigen receptor for the selective treatment of Tcell malignancies. Blood. 2015;126:983-92.

40. Frankel AE, Laver JH, Willingham MC, Burns LJ, Kersey JH, Vallera DA. Therapy of patients with T-cell lymphomas and leukemias using an anti-CD7 monoclonal antibody-ricin A chain immunotoxin. Leuk Lymphoma. 1997;26:287-98.

41. Lee DM, Staats HF, Sundy JS, Patel DD, Sempowski GD, Scearce $\mathrm{RM}$, et al. Immunologic characterization of CD7-deficient mice. J Immunol. 1998;160:5749-56.

42. Subrahmanyam G, Rudd CE, Schneider H. Association of T cell antigen CD7 with type II phosphatidylinositol-4 kinase, a key component in pathways of inositol phosphate turnover. Eur $\mathbf{J}$ Immunol. 2003;33:46-52.

43. Lyman SD, Escobar S, Rousseau AM, Armstrong A, Fanslow WC. Identification of $\mathrm{CD} 7$ as a cognate of the human K12 (SECTM1) protein. J Biol Chem. 2000;275:3431-7.

44. Wang T, Huang C, Lopez-Coral A, Slentz-Kesler KA, Xiao M, Wherry EJ, et al. K12/SECTM1, an interferon-gamma regulated molecule, synergizes with CD28 to costimulate human T cell proliferation. J Leukoc Biol. 2012;91:449-59.

45. Qasim W, Mackey T, Sinclair J, Chatziandreou I, Kinnon C, Thrasher AJ, et al. Lentiviral vectors for T-cell suicide gene therapy: preservation of T-cell effector function after cytokinemediated transduction. Mol Ther. 2007;15:355-60.

46. Afgan E, Baker D, Batut B, van den Beek M, Bouvier D, Cech M, et al. The Galaxy platform for accessible, reproducible and collaborative biomedical analyses: 2018 update. Nucleic Acids Res. 2018;46:W537-W44.

47. Ye K, Schulz MH, Long Q, Apweiler R, Ning Z. Pindel: a pattern growth approach to detect break points of large deletions and medium sized insertions from paired-end short reads. Bioinformatics. 2009;25:2865-71. 